\title{
Stochastic resonance in stochastic PDEs
}

\author{
Nils Berglund and Rita Nader
}

\begin{abstract}
We consider stochastic partial differential equations (SPDEs) on the one-dimensional torus, driven by space-time white noise, and with a time-periodic drift term, which vanishes on two stable and one unstable equilibrium branches. Each of the stable branches approaches the unstable one once per period. We prove that there exists a critical noise intensity, depending on the forcing period and on the minimal distance between equilibrium branches, such that the probability that solutions of the SPDE make transitions between stable equilibria is exponentially small for subcritical noise intensity, while they happen with probability exponentially close to 1 for supercritical noise intensity. Concentration estimates of solutions are given in the $H^{s}$ Sobolev norm for any $s<\frac{1}{2}$. The results generalise to an infinite-dimensional setting those obtained for 1-dimensional SDEs in [5].
\end{abstract}

Date. July 15, 2021. Updated August 9, 2021.

2020 Mathematical Subject Classification. 60H15, 60G17 (primary), 34F15, 37H20 (secondary)

Keywords and phrases. Stochastic PDEs, stochastic resonance, sample-path estimates, slow-fast systems, transcritical bifurcation.

\section{Introduction}

Stochastic resonance can occur when a bistable or multistable dynamical system is forced periodically in time, while also subjected to noise. When the forcing period is close to the typical time needed by the noise to move the system from one metastable state to another one, large-amplitude, nearly periodic oscillations may occur. Even if this resonance condition is not exactly met, the response of the system shows a trace of the periodic forcing in its power spectrum.

The mechanism of stochastic resonance was initially introduced in the context of climate science [23, 2], to propose an explanation for the relation between Milankovitch cycles and glacial periods. Since then, stochastic resonance has shown up in several other applications to ecology and climate science, see for instance [25, 12, 1]. It also appears in many other applications, including neuroscience [22] and quantum electronics [27]. We refer to [26, 16, 18] for comprehensive reviews on this topic.

The most precise mathematical results on stochastic resonance have been obtained for onedimensional stochastic differential equations (SDEs) of the form

$$
\mathrm{d} X_{t}=f\left(\varepsilon t, X_{t}\right) \mathrm{d} t+\sigma \mathrm{d} W_{t}
$$

where $W_{t}$ is a standard Wiener process, and $f$ is a time-periodic bistable drift term. A standard example is

$$
f(\varepsilon t, x)=x-x^{3}+A \cos (\varepsilon t)=-\frac{\partial}{\partial x}\left(\frac{1}{4} x^{4}-\frac{1}{2} x^{2}-A \cos (\varepsilon t) x\right) .
$$


Whenever $A$ is smaller than a critical value given by $A_{\mathrm{c}}=\frac{2}{3 \sqrt{3}}$, the drift term vanishes in three different values of $x$, which correspond to equilibrium states of the system with a frozen value of $\varepsilon t$. These states are also critical points of the double-well potential $V(x, \varepsilon t)=\frac{1}{4} x^{4}-\frac{1}{2} x^{2}-A \cos (\varepsilon t) x$, where the middle point is the unstable saddle, and the two outer points are stable potential minima.

The first investigations of stochastic resonance in systems of the form (1.1) focused on the case of small amplitude $A[14,15,21]$, but many other parameter regimes have been considered as well (see [19] for an overview of mathematical results). Here, we will be mainly interested in the case where $A$ is slightly smaller than $A_{\mathrm{c}}$, which was analysed in the one-dimensional setting in the work [5]. In that situation, one can prove that there exists a critical noise intensity $\sigma_{\mathrm{c}}$ such that when $\sigma \ll \sigma_{\mathrm{c}}$, transitions between potential minima are very rare, while for $\sigma \gg \sigma_{\mathrm{c}}$, it is very likely that the system goes back and forth between the local minima twice per period.

The present work is concerned with a generalisation of (1.1) to the infinite-dimensional setting. We will consider stochastic partial differential equations (SPDEs) of the form

$$
\mathrm{d} \phi(t, x)=[\Delta \phi(t, x)+f(\varepsilon t, \phi(t, x))] \mathrm{d} t+\sigma \mathrm{d} W(t, x),
$$

where $x$ belongs to the one-dimensional torus $\mathbb{T}=\mathbb{R} / L \mathbb{Z}$, and $W(t, x)$ denotes space-time white noise given by a cylindrical Wiener process. The drift term $f$ is again assumed to describe a bistable situation. For instance, the choice (1.2) corresponds to a periodically forced Allen-Cahn equation. Our results apply, however, to more general drift terms $f$, that only need to satisfy a number of regularity and growth conditions.

The analysis requires an extension to the infinite-dimensional situation of SPDEs of samplepath methods introduced in [4,5] for the one-dimensional setting, and extended in [6, 7] to arbitrary finite dimensions. A first step towards extending those methods to infinite dimensions has been taken in [17]. However, that work considers noise that is coloured in space and white in time, given by a $Q$-Wiener process with trace class covariance, while we consider here the more difficult situation of space-time white noise.

Our main results can be summarised as follows. As above, we assume that the time-periodic drift term $f$ vanishes on three branches, two of which come close to each other or meet once per period. The minimal distance between the branches at these close encounters is measured by a small parameter $\delta$, which corresponds to $A_{\mathrm{c}}-A$ in the particular case where $f$ is given by (1.2). We then have the following results.

- Theorem 2.4 states that as long as the equilibrium branches are well-separated, solutions of the SPDE (1.3) are likely to remain close to deterministic solutions tracking the stable branches. Closeness is measured in the $H^{s}$ Sobolev norm, where $s$ is strictly smaller than $\frac{1}{2}$, but can be arbitrarily close to $\frac{1}{2}$.

- When equilibrium branches become close to each other, we decompose the solution $\phi(t, x)$ into its spatial mean $\phi_{0}(t)$, and its zero-mean transverse part $\phi_{\perp}(t, x)$. Theorem 2.9 says that the conclusion of Theorem 2.4 remains valid at bifurcation points for the transverse part.

- The behaviour of the spatial mean $\phi_{0}(t)$ depends on the value of the noise intensity $\sigma$. Theorem 2.10 implies that in the weak-noise regime $\sigma \ll \sigma_{\mathrm{c}}=(\delta \vee \varepsilon)^{3 / 4}$, sample paths are still likely to remain close to the same stable equilibrium. The probability of making a transition to the other stable equilibrium is exponentially small in $\sigma_{\mathrm{c}}^{2} / \sigma^{2}$.

- In the strong-noise regime $\sigma \geqslant \sigma_{\mathrm{c}}=(\delta \vee \varepsilon)^{3 / 4}$, transitions between equilibrium branches become more likely. Theorem 2.11 implies that the probability not to make a transition to the other stable equilibrium when approaching an avoided bifurcation point decays roughly like $\exp \left[-\sigma^{4 / 3} /\left(\varepsilon \log \left(\sigma^{-1}\right)\right)\right]$. 
Our results thus show that similarly to the one-dimensional situation considered in [5], depending on the noise intensity, transitions between stable equilibria are either exponentially rare, or happen with a probability exponentially close to 1 . There are some differences in the error terms, which are due to the fact that we have to deal with the transverse part $\phi_{\perp}$ of the solution.

The main difficulty of the analysis comes from the fact that we work with space-time white noise in an infinite-dimensional situation. This prevents us from applying directly the methods from [6], which work in finite dimension, and include dimension-dependent error terms. These error estimates can be adapted to trace class noise, as was done in [17], but the white noise case needs a different approach, relying on more careful estimates in various Sobolev norms. Key results are an estimate for a linearised equation based on the Fourier decomposition, presented in Section 3.2. and a Schauder estimate given in Lemma 3.5.

The remainder of this paper is organised as follows. In Section 2, we give the precise assumptions on the SPDEs we consider, and all main results, as well as a discussion of the different parameter regimes. Section 3 contains the proofs for the stable case, that is, as long as the system does not approach any bifurcation points, while Section 4 contains the proofs for the cases with (avoided) bifurcations. Appendix A recalls several inequalities involving products in Sobolev spaces that are used in the analysis.

\section{Notations}

The system studied in this work depends on three small parameters $\varepsilon, \sigma$ and $\delta$. We write $X \lesssim Y$ to indicate that $X \leqslant c Y$ for a constant $c$ independent of $\varepsilon, \sigma$ and $\delta$, as long as these parameters are small enough. The notation $X \asymp Y$ indicates that one has both $X \lesssim Y$ and $Y \lesssim X$, while Landau's notation $X=\mathcal{O}(Y)$ means that $|X| \lesssim Y$. If $a, b \in \mathbb{R}, a \wedge b$ denotes the minimum of $a$ and $b$, and $a \vee b$ denotes the maximum of $a$ and $b$. Finally, we write $1_{\mathcal{D}}(x)$ for the indicator function of a set or event $\mathcal{D}$.

\section{Acknowledgments}

This work is supported by the ANR project PERISTOCH, ANR-19-CE40-0023. The authors thank Gérard Bourdaud for drawing their attention to the reference [10].

\section{Main results}

\subsection{The set-up}

Let $L, T>0$ be real parameters. We will consider time-dependent SPDEs on the torus $\mathbb{T}=\mathbb{R} / L \mathbb{Z}$ of the form

$$
\mathrm{d} \phi(t, x)=[\Delta \phi(t, x)+f(\varepsilon t, \phi(t, x))] \mathrm{d} t+\sigma \mathrm{d} W(t, x),
$$

for the unknown $\phi: I \times \mathbb{T} \rightarrow \mathbb{R}$, where $I=[0, T]$. Here

- $\varepsilon>0$ is a small parameter quantifying the slow time dependence;

- $\sigma>0$ is a small parameter measuring the noise intensity;

- $f:[0, T] \times \mathbb{R} \rightarrow \mathbb{R}$ is a forcing term satisfying a number of assumptions given below;

- $\mathrm{d} W(t, x)$ denotes space-time white noise on $\mathbb{R}_{+} \times \mathbb{T}$.

Our results extend naturally to the case where $f: \mathbb{R} \times \mathbb{R} \rightarrow \mathbb{R}$ is periodic in the time variable, with period $T$. 
It will be more convenient to work with slow time $\varepsilon t$. Scaling time by a factor $\varepsilon$ yields the equation

$$
\mathrm{d} \phi(t, x)=\frac{1}{\varepsilon}[\Delta \phi(t, x)+f(t, \phi(t, x))] \mathrm{d} t+\frac{\sigma}{\sqrt{\varepsilon}} \mathrm{d} W(t, x) .
$$

It will sometimes be useful to work with a potential $U$ associated with $f$, satisfying

$$
f(t, \phi)=-\partial_{\phi} U(t, \phi) .
$$

The following assumption on the behaviour of $U$ for large values of $\phi$ will be assumed to hold throughout this work.

Assumption 2.1 (Global behaviour of the drift term). The potential $U$ admits, for all $(t, \phi) \in I \times \mathbb{R}$, a decomposition

$$
U(t, \phi)=P(t, \phi)+g(t, \phi)
$$

into a polynomial part and a bounded part. More precisely,

- there exists an integer $p_{0} \geqslant 1$ such that the map $\phi \mapsto P(t, \phi)$ is a polynomial of degree $2 p_{0}$, of the form

$$
P(t, \phi)=\sum_{j=0}^{2 p_{0}} A_{j}(t) \phi^{j}
$$

with coefficients $A_{j} \in \mathcal{C}^{1}(I, \mathbb{R})$ such that $\left|A_{j}(t)\right|$ and $\left|A_{j}^{\prime}(t)\right|$ are bounded uniformly, and $A_{2 p_{0}}(t)>0$ for all $t \in I$;

- the function $g \in \mathcal{C}^{2}(I \times \mathbb{R}, \mathbb{R})$ satisfies

$$
\left|g(t, \phi) \phi^{-1}\right|,\left|\partial_{\phi} g(t, \phi)\right|,\left|\partial_{\phi \phi} g(t, \phi)\right|,\left|\partial_{t} g(t, \phi)\right| \leqslant M
$$

for all $(t, \phi) \in I \times \mathbb{R}$ and some constant $M>0$.

\subsection{The stable case}

We start by considering the case where the drift term $f$ admits a stable equilibrium branch, in the following sense.

Assumption 2.2 (Stable case). There exists a map $\phi^{*}: I \rightarrow \mathbb{R}$ such that

$$
f\left(t, \phi^{*}(t)\right)=0 \quad \forall t \in I .
$$

Furthermore, the linearisation $a(t)=\partial_{\phi} f\left(t, \phi^{*}(t)\right)$ satisfies

$$
-a_{+} \leqslant a(t) \leqslant-a_{-} \quad \forall t \in I
$$

for some constant $a_{ \pm}>0$.

Consider first the deterministic equation

$$
\mathrm{d} \phi(t, x)=\frac{1}{\varepsilon}[\Delta \phi(t, x)+f(t, \phi(t, x))] \mathrm{d} t .
$$


It will be convenient to work with the orthonormal Fourier basis $\left\{e_{k}\right\}_{k \in \mathbb{Z}}$ of $L^{2}(\mathbb{T}, \mathbb{R})$, given by

$$
e_{k}(x)= \begin{cases}\sqrt{\frac{2}{L}} \cos \left(\frac{k \pi x}{L}\right) & \text { if } k>0, \\ \frac{1}{\sqrt{L}} & \text { if } k=0, \\ \sqrt{\frac{2}{L}} \sin \left(\frac{k \pi x}{L}\right) & \text { if } k<0 .\end{cases}
$$

Given a real number $s>0$ and a function $\phi \in L^{2}(\mathbb{T})$ with Fourier expansion

$$
\phi(x)=\sum_{k \in \mathbb{Z}} \phi_{k} e_{k}(x),
$$

we define the fractional Sobolev norm of $\phi$ by

$$
\|\phi\|_{H^{s}}^{2}=\sum_{k \in \mathbb{Z}}\langle k\rangle^{2 s} \phi_{k}^{2}
$$

where we use the "Japanese bracket" notation $\langle k\rangle=\left(1+k^{2}\right)^{1 / 2}$. We denote by $H^{s}=H^{s}(\mathbb{T}, \mathbb{R})$ the fractional Sobolev space (or Bessel potential space) of functions $\phi: \mathbb{T} \rightarrow \mathbb{R}$ admitting a finite $H^{s}$-norm. We then have the following result, which generalises to our infinite-dimensional setting results from singular perturbation theory that are well-known in finite dimension (see in particular [24, 13]).

Proposition 2.3 (Deterministic dynamics in the stable case). There exist constants $C, \varepsilon_{0}>0$ such that for $0<\varepsilon<\varepsilon_{0}$, the equation (2.3) admits a particular solution $\bar{\phi}(t, x)$ satisfying

$$
\left\|\bar{\phi}(t, \cdot)-\phi^{*}(t) e_{0}\right\|_{H^{1}} \leqslant C \varepsilon \quad \forall t \in I .
$$

In the finite-dimensional case, it is known (see [4, Theorem 2.4]) that solutions of the stochastic equation (2.1), starting near the equilibrium branch $\phi^{*}$, remain close to that branch with high probability. To quantify this in our infinite-dimensional situation, given $s>0$ we define for any $h>0$ the set

$$
\mathcal{B}(h)=\left\{(t, \phi): t \in I,\|\phi-\bar{\phi}(t, \cdot)\|_{H^{s}}<h\right\} .
$$

Given an initial condition $\left(0, \phi_{0}\right)$ in $\mathcal{B}(h)$, the first-exit time from $\mathcal{B}(h)$ is the stopping time

$$
\begin{aligned}
\tau_{\mathcal{B}(h)} & =\inf \{t>0:(t, \phi(t, \cdot)) \notin \mathcal{B}(h)\} \\
& =\inf \left\{t>0:\|\phi-\bar{\phi}(t, \cdot)\|_{H^{s}} \geqslant h\right\} .
\end{aligned}
$$

By convention, we set $\tau_{\mathcal{B}(h)}=+\infty$ whenever $(t, \phi(t, \cdot)) \in \mathcal{B}(h)$ for all $t \in I$.

Theorem 2.4 (Stochastic dynamics in the stable case). For any $s \in\left(0, \frac{1}{2}\right)$ and any $\nu>0$, there exist constants $\kappa=\kappa(s), \varepsilon_{0}, h_{0}$ and $C(\kappa, t, \varepsilon, s)>0$ such that, whenever $0<\varepsilon \leqslant \varepsilon_{0}$ and $0<h \leqslant h_{0} \varepsilon^{\nu}$, the solution of (2.1) with initial condition $\phi(0, \cdot)=\bar{\phi}(0, \cdot)$ satisfies

$$
\mathbb{P}\left\{\tau_{\mathcal{B}(h)}<t\right\} \leqslant C(\kappa, t, \varepsilon, s) \exp \left\{-\kappa \frac{h^{2}}{\sigma^{2}}\left[1-\mathcal{O}\left(\frac{h}{\varepsilon^{\nu}}\right)\right]\right\} .
$$

for all $t \in I$. 
Remark 2.5. The proof yields explicit bounds on $C(\kappa, t, \varepsilon, s)$. In particular, this quantity can be taken proportional to $t / \varepsilon$, while its dependence on $\kappa$ and $s$ is more complicated.

Remark 2.6. The result also holds for general initial conditions $\phi(0, \cdot)$ in an $H^{s}$-neighbourhood of order 1 of $\bar{\phi}(0, \cdot)$, provided one only considers the probability of leaving $\mathcal{B}(h)$ after a time of order $\varepsilon \log \left(\|\phi(0, \cdot)\|_{H^{s}} h^{-1}\right)$, since solutions need a time of that order to reach $\mathcal{B}(h)$. See [7, Theorem 5.1.6] for a precise formulation, which can be adapted to the present situation by a similar argument.

\subsection{Bifurcations and avoided bifurcations}

We now proceed to stating the main part of our results, which deal with systems admitting bifurcations or avoided bifurcations. As a motivating example, consider again the periodically forced Allen-Cahn equation

$$
\mathrm{d} \phi(t, x)=\frac{1}{\varepsilon}\left[\Delta \phi(t, x)+\phi(t, x)-\phi(t, x)^{3}+A \cos (t)\right] \mathrm{d} t+\frac{\sigma}{\sqrt{\varepsilon}} \mathrm{d} W(t, x) .
$$

Whenever $A<A_{\mathrm{c}}=\frac{2}{3 \sqrt{3}}$, the equation $\phi-\phi^{3}+A \cos (t)=0$ has exactly three solutions

$$
\phi_{1}^{*}(t)<\phi_{2}^{*}(t)<\phi_{3}^{*}(t) .
$$

If $t$ is replaced by a fixed parameter $t_{0}$, the equilibrium branches $\phi_{1,3}^{*}\left(t_{0}\right)$ are stable for the deterministic fast system

$$
\partial_{t} \phi(t, x)=\Delta \phi(t, x)+\phi(t, x)-\phi(t, x)^{3}+A \cos \left(t_{0}\right),
$$

while $\phi_{2}^{*}\left(t_{0}\right)$ is unstable. If $A=A_{\mathrm{c}}$, a stable branch and the unstable branch meet a transcritical bifurcation point whenever $t$ is a multiple of $\pi$. If $A$ is slightly smaller than $A_{\mathfrak{c}}$, the branches approach each other without quite touching. However, noise may trigger transitions between the branches, which is one of the basic mechanisms responsible for stochastic resonance.

We will consider more general equations of the form (2.1), assuming that the drift term $f(t, \phi)$ vanishes on three equilibrium branches, two of which come close to each other at particular times. Whenever the three branches are well-separated, the dynamics near stable branches can be described by Theorem 2.4. It is thus sufficient to describe the dynamics near times of bifurcation, or avoided bifurcation. By an affine change of variables, it is always possible to translate these (avoided) bifurcation points to the origin $(t, \phi)=(0,0)$. We will then make the following assumptions.

Assumption 2.7 (Bifurcation point). The drift term $f$ is of class $\mathcal{C}^{3}$, and satisfies

$$
\begin{aligned}
f(t, 0) & =\delta+a_{1} t^{2}+\mathcal{O}\left(t^{3}\right), \\
\partial_{\phi} f(t, 0) & =\mathcal{O}\left(t^{2}\right), \\
\partial_{\phi \phi} f(0,0) & <0
\end{aligned}
$$

for constants $\delta \geqslant 0$ and $a_{1}>0$.

Scaling time, space and $\phi$ appropriately, one can always assume that $a_{1}=1$ (see Section 4). Under Assumption 2.7, one can check (see [5, Section 4]) that in a neighbourhood of $(0,0)$, the drift term $f(t, \phi)$ vanishes only on two branches $\phi_{ \pm}^{*}(t)$, satisfying

$$
\begin{aligned}
\phi_{ \pm}^{*}(t) & \asymp \pm(\sqrt{\delta}+|t|), \\
a_{ \pm}(t)=\partial_{\phi} f\left(t, \phi_{ \pm}^{*}(t)\right) & \asymp \mp(\sqrt{\delta}+|t|) .
\end{aligned}
$$




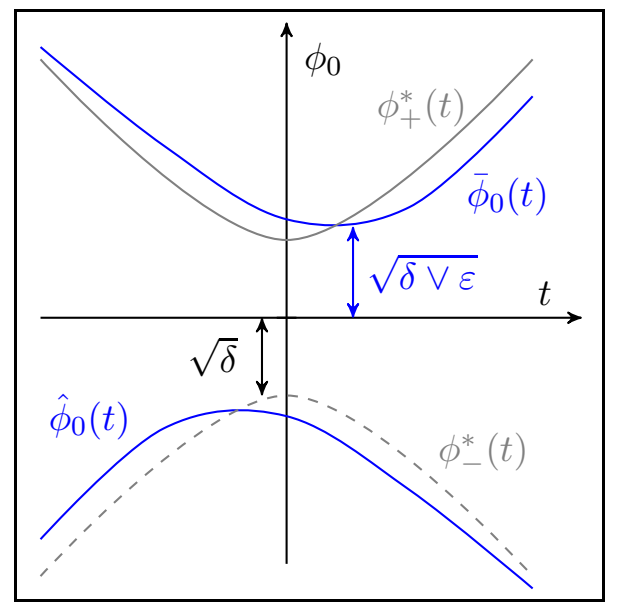

FIGURE 1. Equilibrium branches and associated adiabatic solutions near the avoided bifurcation point $(0,0)$.

In particular, $\phi_{+}^{*}$ is stable, while $\phi_{-}^{*}$ is unstable, unless $\delta=0$ and $t=0$, when there is a transcritical bifurcation (Figure 1).

In what follows, we will rewrite the SPDE (2.1) in the form

$$
\mathrm{d} \phi(t, x)=\frac{1}{\varepsilon}\left[\Delta \phi(t, x)+g(t)-\phi(t, x)^{2}-b(t, \phi(t, x))\right] \mathrm{d} t+\frac{\sigma}{\sqrt{\varepsilon}} \mathrm{d} W(t, x),
$$

where

$$
\begin{aligned}
g(t) & =\delta+t^{2}+\mathcal{O}\left(t^{3}\right), \\
b(t, \phi) & =\mathcal{O}\left(\phi^{3}\right)+\mathcal{O}\left(t \phi^{2}\right)+\mathcal{O}\left(t^{2} \phi\right) .
\end{aligned}
$$

It will be convenient to decompose the solution of (2.4) into its spatial mean and oscillating part, by writing

$$
\phi(t, x)=\phi_{0}(t) e_{0}(x)+\phi_{\perp}(t, x), \quad \int_{\mathbb{T}} \phi_{\perp}(t, x) \mathrm{d} x=0 .
$$

One then finds that the SPDE (2.4) is equivalent to the coupled SDE-SPDE system

$$
\begin{aligned}
\mathrm{d} \phi_{0}(t) & =\frac{1}{\varepsilon}\left[g(t)-\phi_{0}(t)^{2}-b\left(t, \phi_{0}(t) e_{0}\right)+b_{0}\left(t, \phi_{0}(t), \phi_{\perp}(t, \cdot)\right)\right] \mathrm{d} t+\frac{\sigma}{\sqrt{\varepsilon}} \mathrm{d} W_{0}(t), \quad \text { (2.6) } \\
\mathrm{d} \phi_{\perp}(t, x) & =\frac{1}{\varepsilon}\left[\Delta \phi_{\perp}(t, x)+a\left(t, \phi_{0}(t)\right) \phi_{\perp}(t, x)+b_{\perp}\left(t, \phi_{0}(t), \phi_{\perp}(t, \cdot)\right)\right] \mathrm{d} t+\frac{\sigma}{\sqrt{\varepsilon}} \mathrm{d} W_{\perp}(t, x),
\end{aligned}
$$

where $W_{0}(t)$ is a standard Brownian motion, $W_{\perp}(t, x)$ is an independent zero-mean space-time white noise,

$$
a\left(t, \phi_{0}\right)=-2 \phi_{0}-\frac{1}{\sqrt{L}} \partial_{\phi} b\left(t, \phi_{0} e_{0}\right),
$$

while $b_{0}$ and $b_{\perp}$ are (non-local) remainders specified in (4.1) below.

Proposition 2.8 (Deterministic dynamics near the origin). The deterministic equation given by (2.4) with $\sigma=0$ admits a particular solution satisfying $\phi_{\perp}(t, x)=0$, while $\phi_{0}$ obeys the ordinary differential equation

$$
\varepsilon \dot{\phi}_{0}(t)=g(t)-\phi_{0}(t)^{2}-b\left(t, \phi_{0}(t) e_{0}\right) .
$$


The equation (2.7) for $\phi_{0}(t)$ is exactly of the form previously analysed in the work [5]. In particular, Theorem 2.5 in that article states that there exists a particular solution $\bar{\phi}_{0}(t)$ tracking $\phi_{+}^{\star}(t)$, in the sense that there are constants $T_{0}, c_{0}>0$ such that

$$
\bar{\phi}_{0}(t)-\phi_{+}^{*}(t) \asymp \begin{cases}\frac{\varepsilon}{|t|} & \text { for }-T_{0} \leqslant t \leqslant-c_{0}(\sqrt{\delta \vee \varepsilon}), \\ -\frac{\varepsilon}{|t|} & \text { for } c_{0} \sqrt{\delta \vee \varepsilon} \leqslant t \leqslant T_{0}\end{cases}
$$

(Figure 1). Furthermore, one has

$$
\bar{\phi}_{0}(t) \asymp \sqrt{\delta \vee \varepsilon} \quad \text { for }|t| \leqslant c_{0} \sqrt{\delta \vee \varepsilon} .
$$

As a consequence, the linearisation

$$
\begin{aligned}
\bar{a}\left(t, \bar{\phi}_{0}(t)\right) & =\left.\partial_{\phi}\left[g(t)-\phi^{2}-b(t, \phi)\right]\right|_{\phi=\bar{\phi}_{0}(t)} \\
& =-2 \bar{\phi}_{0}(t)-\partial_{\phi} b\left(t, \bar{\phi}_{0}(t)\right)
\end{aligned}
$$

satisfies

$$
\bar{a}\left(t, \bar{\phi}_{0}(t)\right) \asymp-(|t| \vee \sqrt{\delta \vee \varepsilon})
$$

for all $t \in\left[-T_{0}, T_{0}\right]$. By a symmetry argument, similar results, with some signs reversed, hold for a particular solutions $\hat{\phi}_{0}(t)$ tracking the unstable equilibrium branch $\phi_{-}^{*}(t)$.

Let $\zeta(t)$ be the solution of

$$
\varepsilon \dot{\zeta}(t)=2 \bar{a}\left(t, \bar{\phi}_{0}(t)\right) \zeta(t)+1
$$

with initial condition $\zeta\left(-T_{0}\right)=\left(2\left|\bar{a}\left(-T_{0}, \bar{\phi}_{0}\left(-T_{0}\right)\right)\right|\right)^{-1}$. This function is related to the variance of the linearisation around $\bar{\phi}_{0}(t)$ of the equation for $\phi_{0}$. It can be written explicitly as

$$
\begin{aligned}
\zeta(t) & =\frac{1}{2\left|\bar{a}\left(-T_{0}, \bar{\phi}_{0}\left(-T_{0}\right)\right)\right|} \mathrm{e}^{2 \bar{\alpha}\left(t,-T_{0}\right) / \varepsilon}+\frac{1}{\varepsilon} \int_{-T_{0}}^{t} \mathrm{e}^{2 \bar{\alpha}\left(t, t_{1}\right) / \varepsilon} \mathrm{d} t_{1}, \\
\bar{\alpha}\left(t, t_{1}\right) & =\int_{t_{1}}^{t} \bar{a}\left(u, \bar{\phi}_{0}(u)\right) \mathrm{d} u .
\end{aligned}
$$

However, it is more important for what follows to know that

$$
\zeta(t) \asymp \frac{1}{\left|\bar{a}\left(t, \bar{\phi}_{0}(t)\right)\right|} \asymp \frac{1}{|t| \vee \sqrt{\delta \vee \varepsilon}} \quad \forall t \in\left[-T_{0}, T_{0}\right],
$$

see [5, Equation (4.18)]. With these notations in place, we are able to define the sets

$$
\begin{aligned}
\mathcal{B}_{0}(h) & =\left\{\left(t, \phi_{0}\right): t \in\left[-T_{0}, T_{0}\right],\left|\phi_{0}-\bar{\phi}_{0}(t)\right|<h \sqrt{\zeta(t)}\right\}, \\
\mathcal{B}_{\perp}\left(h_{\perp}\right) & =\left\{(t, \phi): t \in\left[-T_{0}, T_{0}\right],\left\|\phi_{\perp}\right\|_{H^{s}}<h_{\perp}\right\},
\end{aligned}
$$

where $s \in\left(0, \frac{1}{2}\right)$, and $h, h_{\perp}>0$. The exit from $\mathcal{B}_{\perp}\left(h_{\perp}\right)$ is described by the following analogue of Theorem 2.4. 


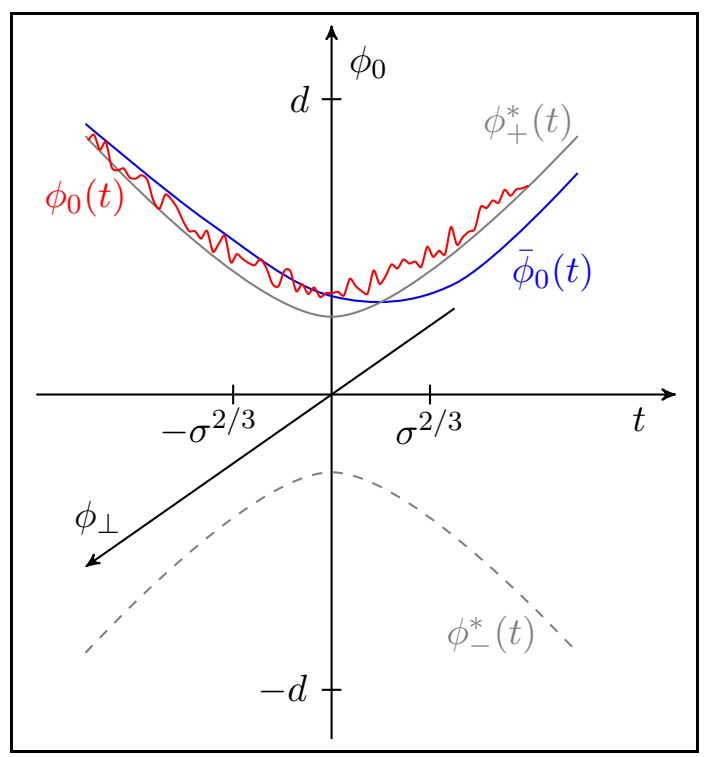

FIGURE 2. Weak noise regime $\sigma \ll(\delta \vee \varepsilon)^{3 / 4}$. The equilibrium branches $\phi_{ \pm}^{*}(t)$, as well as the deterministic solution $\bar{\phi}_{0}(t)$, belong to the hyperplane $\left\{\phi_{\perp}=0\right\}$, while $\phi_{0}(t)$ denotes the projection of the solution $\phi(t, x)$ on this hyperplane.

Theorem 2.9 (Transverse stochastic dynamics for $\phi_{\perp}$ ). If $T_{0}$ is sufficiently small, then for any $s \in\left(0, \frac{1}{2}\right)$ and any $\nu>0$, there exist constants $\kappa=\kappa(s), \varepsilon_{0}, h_{\perp}^{0}$ and $C(\kappa, t, \varepsilon, s)>0$ such that, whenever $0<\varepsilon \leqslant \varepsilon_{0}$ and $0<h_{\perp} \leqslant h_{\perp}^{0} \varepsilon^{\nu}$, the solution of (2.4) with initial condition $\phi\left(-T_{0}, \cdot\right)=\bar{\phi}_{0}\left(-T_{0}\right) e_{0}$ satisfies

$$
\mathbb{P}\left\{\tau_{\mathcal{B}_{\perp}\left(h_{\perp}\right)}<t \wedge \tau_{\mathcal{B}_{0}(h)}\right\} \leqslant C(\kappa, t, \varepsilon, s) \exp \left\{-\kappa \frac{h_{\perp}^{2}}{2 \sigma^{2}}\left[1-\mathcal{O}\left(\frac{h_{\perp}}{\varepsilon^{\nu}}\right)\right]\right\} .
$$

The result remains true when $\tau_{\mathcal{B}_{0}(h)}$ is replaced by $\inf \left\{t \in\left[-T_{0}, T_{0}\right]:\left|\phi_{0}(t)\right|>d\right\}$ for any sufficiently small d of order 1 .

As before, the result also holds for initial conditions with a transverse part $\phi_{\perp}\left(-T_{0}, \cdot\right)$ having $H^{s}$ norm up to order 1 , provided one considers the probability of leaving $\mathcal{B}_{\perp}\left(h_{\perp}\right)$ after a time of order $\left.\varepsilon \log \left(\left\|\phi_{\perp}\left(-T_{0}, \cdot\right)\right\|_{H^{1}} h_{\perp}^{-1}\right)\right)$.

On the other hand, the exit from $\mathcal{B}_{0}(h)$ is described by the following result.

Theorem 2.10 (Stochastic dynamics near $\bar{\phi}_{0}(t)$ ). For any $t \in\left[-T_{0}, T_{0}\right]$, let

$$
\hat{\zeta}(t)=\sup _{-T_{0} \leqslant s \leqslant t} \zeta(s) .
$$

Then there exist constants $\varepsilon_{0}, h_{0}, c_{\perp}, \kappa>0$ such that, whenever $0<\varepsilon \leqslant \varepsilon_{0}, 0<h \leqslant h_{0} \hat{\zeta}(t)^{-3 / 2}$ and $0<h_{\perp}<c_{\perp} h \hat{\zeta}(t)^{1 / 2}$, the solution of (2.4) with initial condition $\phi\left(-T_{0}, \cdot\right)=\bar{\phi}_{0}\left(-T_{0}\right) e_{0}$ satisfies

$$
\mathbb{P}\left\{\tau_{\mathcal{B}_{0}(h)}<t \wedge \tau_{\mathcal{B}_{\perp}\left(h_{\perp}\right)}\right\} \leqslant C(t, \varepsilon) \exp \left\{-\kappa \frac{h^{2}}{2 \sigma^{2}}\right\},
$$

where $\kappa=1-\mathcal{O}\left(h \hat{\zeta}(t)^{3 / 2}\right)$ and $C(t, \varepsilon)=\frac{\bar{\alpha}\left(t,-T_{0}\right)}{\varepsilon^{2}}+2$. As before, the bound extends to general initial conditions in $\mathcal{B}_{\perp}\left(h_{\perp}\right)$ with $\left(-T_{0}, \phi_{0}\left(-T_{0}\right)\right)$ in $\mathcal{B}_{0}(h)$. 


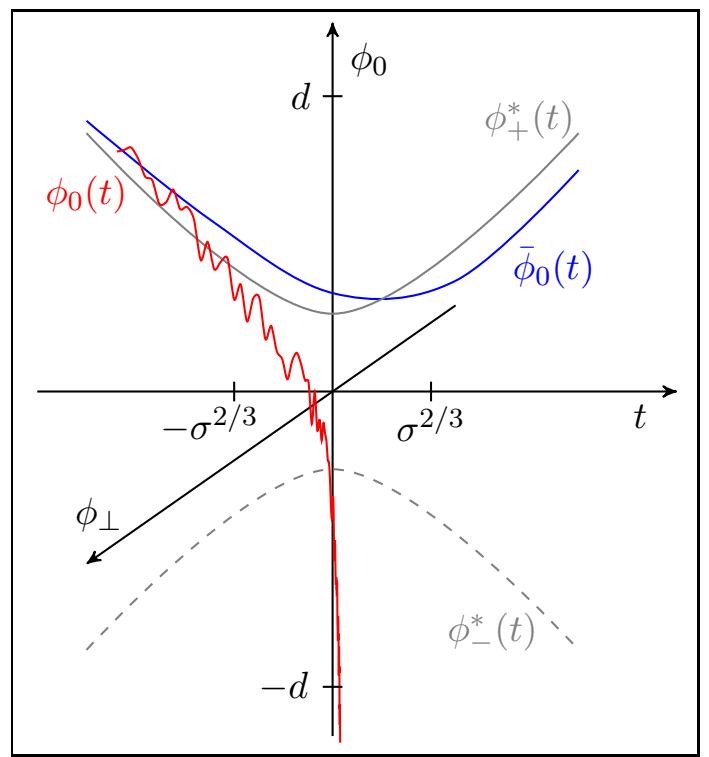

Figure 3. Strong noise regime $\sigma \gg(\delta \vee \varepsilon)^{3 / 4}$. Solutions are likely to cross the unstable equilibrium branch $\phi_{-}^{*}(t)$.

One consequence of this result is that there are two qualitatively different regimes, depending on the noise intensity:

- Weak-noise regime: if $\sigma \ll(\delta \vee \varepsilon)^{3 / 4}$, Theorem 2.10 can be applied for any $t \in\left[-T_{0}, T_{0}\right]$, and shows that $\phi_{0}(t)$ remains close to $\bar{\phi}_{0}(t)$ with high probability during the whole time interval (Figure 2).

- Strong-noise regime: if $\sigma \gg(\delta \vee \varepsilon)^{3 / 4}$, Theorem 2.10 can only be applied up to times $t$ of order $-\sigma^{2 / 3}$, showing that $\phi_{0}(t)$ is unlikely to become negative up to times of that order (Figure 3).

The behaviour in the strong-noise regime for times $t \geqslant-c_{1} \sigma^{2 / 3}$ is described by the following theorem.

Theorem 2.11 (Strong-noise regime). Fix sufficiently small constants $d, c_{1}>0$. Let $h>0$ be such that

$$
\bar{\phi}_{0}(t)+h \sqrt{\zeta(t)} \leqslant d \quad \forall t \in\left[-c_{1} \sigma^{2 / 3}, c_{1} \sigma^{2 / 3}\right] .
$$

Then there exist constants $\kappa, \bar{c}_{\perp}>0$ such that for

$$
0<h_{\perp}<\bar{c}_{\perp}\left[\sigma^{2 / 3} \wedge \sqrt{h} \hat{\zeta}(t)^{-1 / 4}\right]
$$

any solution of (2.4) starting at time $-c_{1} \sigma^{2 / 3}$ with an initial value $\phi_{0}$ belonging to the interval $\left(-d, \bar{\phi}_{0}\left(-c_{1} \sigma^{2 / 3}+\frac{1}{2} h \sqrt{\zeta\left(-c_{1} \sigma^{2 / 3}\right)}\right)\right]$ satisfies

$$
\begin{aligned}
\mathbb{P}\left\{\phi_{0}\left(t_{1}\right)>-d \forall t_{1} \in\left[-c_{1} \sigma^{2 / 3}, t \wedge \tau_{\left.\mathcal{B}_{\perp}\left(h_{\perp}\right)\right\} \leqslant}\right.\right. & \frac{3}{2} \exp \left\{-\kappa \frac{\hat{\alpha}\left(t,-c_{1} \sigma^{2 / 3}\right)}{\varepsilon \log \left(\sigma^{-1}\right)}\right\} \\
& +C(t, \varepsilon) \mathrm{e}^{-\kappa h^{2} / \sigma^{2}}
\end{aligned}
$$

for all $t \in\left[-c_{1} \sigma^{2 / 3}, c_{1} \sigma^{2 / 3}\right]$, where

$$
\hat{\alpha}\left(t, t_{1}\right)=\int_{t_{1}}^{t} \hat{a}\left(t_{2}, \hat{\phi}_{0}\left(t_{2}\right)\right) \mathrm{d} t_{2}, \quad \hat{a}\left(t, \hat{\phi}_{0}(t)\right)=\left.\partial_{\phi}\left[g(t)-\phi^{2}+b(t, \phi)\right]\right|_{\phi=\hat{\phi}_{0}(t)}
$$




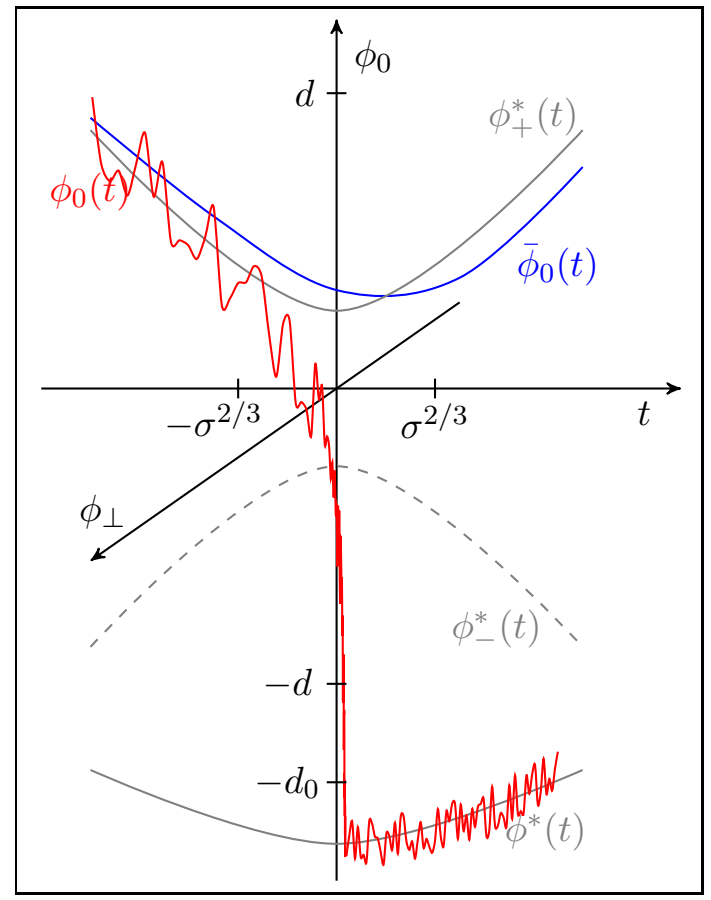

FIgURE 4. Strong noise regime, behaviour after reaching level $-d$. If the drift term is negative, bounded away from zero, in an interval $\left[-d_{0},-d\right]$, solutions are likely to reach $-d_{0}$ after another time of order $\varepsilon$.

and $C(t, \varepsilon)=\frac{\left|\bar{\alpha}\left(t,-c_{1} \sigma^{2 / 3}\right)\right|}{\varepsilon^{2}}+2$.

Remark 2.12. The condition (2.9) is required since we did not make any assumptions on the behaviour of $f$ for $x \geqslant d$. For instance, our results apply if there exist more equilibrium branches above $d$. If, however, there are no such branches, as in the case of the Allen-Cahn equation with drift term (1.2), this condition can probably be relaxed.

To complete the description of the dynamics, we also need to show that once the process has reached level $-d$, it is also likely to reach a neighbourhood of the next stable equilibrium branch, where one can then apply Theorem 2.4 to describe the dynamics up to the next (avoided) bifurcation point (Figure 44). This can be easily done via the following analogue of [5, Proposition 4.7].

Proposition 2.13 (Reaching level $-d_{0}<-d$ ). There exists a constant $M>0$ such that if the drift term $f$ satisfies

$$
f(t, \phi) \leqslant-f_{0}-M h_{\perp}^{2} \quad \forall(t, x) \in\left[-T_{0}, T_{0}\right] \times\left[-d_{0},-d+\rho\right]
$$

for some constants $d_{0}, f_{0}>0$ and $\rho \in(0, d)$, then there exist constant $\tilde{c}, \tilde{\kappa}>0$ such that for all $t_{0} \in\left[-T_{0}, T_{0}-\tilde{c} \varepsilon\right]$, the solution of (2.4) with initial condition $\phi_{0}\left(t_{0}\right)=-d$ satisfies

$$
\mathbb{P}\left\{\phi_{0}\left(t_{1}\right)>-d_{0} \forall t_{1} \in\left[t_{0},\left(t_{0}+\tilde{c} \varepsilon\right) \wedge \tau_{\mathcal{B}_{\perp}}\left(h_{\perp}\right)\right]\right\} \leqslant \mathrm{e}^{-\tilde{\kappa} / \sigma^{2}} .
$$

\subsection{Discussion}

Let us first consider the weak-noise regime $\sigma \ll(\delta \vee \varepsilon)^{3 / 4}$. For Theorems 2.9 and 2.10 to yield useful results, we need

$$
h \gg \sigma, \quad h_{\perp} \gg \sigma .
$$


If $t \geqslant 0$, then $\hat{\zeta}(t) \asymp(\delta \vee \varepsilon)^{-1 / 2}$. For the theorems to be applicable, we then need the conditions

$$
h \lesssim(\delta \vee \varepsilon)^{3 / 4}, \quad h_{\perp} \lesssim h(\delta \vee \varepsilon)^{-1 / 4} \wedge \varepsilon^{\nu},
$$

where $\nu>0$ can be chosen arbitrarily small. The weak-noise condition implies that all conditions on $h$ and $h_{\perp}$ can indeed be met simultaneously. In particular, since the minimal value of $\bar{\phi}_{0}(t)$ has $\operatorname{order}(\delta \vee \varepsilon)^{1 / 2}$, we can take $h$ of order $(\delta \vee \varepsilon)^{3 / 4}$, and $h_{\perp}$ of order $(\delta \vee \varepsilon)^{1 / 2} \wedge \varepsilon^{\nu}$. We thus obtain

$$
\begin{aligned}
\mathbb{P}\left\{\exists t \in\left[-T_{0}, T_{0}\right]: \phi_{0}(t)<0\right\} & \leqslant \mathbb{P}\left\{\tau_{\mathcal{B}_{0}(h)} \wedge \tau_{\left.\mathcal{B}_{\perp}\left(h_{\perp}\right)<T_{0}\right\}}\right. \\
& =\mathbb{P}\left\{\tau_{\mathcal{B}_{\perp}\left(h_{\perp}\right)}<T_{0} \wedge \tau_{\mathcal{B}_{0}(h)}\right\}+\mathbb{P}\left\{\tau_{\mathcal{B}_{0}(h)}<T_{0} \wedge \tau_{\mathcal{B}_{\perp}\left(h_{\perp}\right)}\right\} \\
& \leqslant C_{1}(\varepsilon) \exp \left\{-\kappa \frac{(\delta \vee \varepsilon)^{3 / 2} \wedge \varepsilon^{2 \nu}}{\sigma^{2}}\right\} .
\end{aligned}
$$

The term $\varepsilon^{2 \nu}$ can be disregarded as soon as $\delta$ is sufficiently small. In other words, the probability of making a transition from a neighbourhood of the stable branch $\phi_{+}^{*}$ to the unstable branch $\phi_{-}^{*}$ or to the other stable branch is exponentially small, with a parameter of order $(\delta \vee \varepsilon)^{3 / 2} / \sigma^{2}$.

Consider now the strong-noise regime $\sigma \geqslant(\delta \vee \varepsilon)^{3 / 4}$. We still require the conditions (2.11) to hold, but modify the upper bounds on $h$ and $h_{\perp}$. As long as $t<-c_{1} \sigma^{2 / 3}$, Theorem 2.10 can be applied with $h \lesssim|t|^{3 / 2}$, yielding

$$
\mathbb{P}\left\{\tau_{\mathcal{B}_{0}(h)} \wedge \tau_{\mathcal{B}_{\perp}\left(h_{\perp}\right)}<t\right\} \leqslant C(t, \varepsilon) \exp \left\{-\kappa^{\prime} \frac{|t|^{3}}{\sigma^{2}}\right\}
$$

for some $\kappa^{\prime}>0$. This shows in particular that $\phi_{0}$ is unlikely to reach 0 before times of order $-\sigma^{2 / 3}$.

To see what happens for larger times, we do no longer use Theorem 2.10 , but only Theorems 2.9 and 2.11, applied to an interval of the form $\left[-c_{1} \sigma^{2 / 3},-c_{2} \sigma^{2 / 3}\right]$. Then $\hat{\alpha}\left(-c_{2} \sigma^{2 / 3},-c_{1} \sigma^{2 / 3}\right)$ has order $\sigma^{4 / 3}$ and the conditions on $h$ and $h_{\perp}$ can be summarised as

$$
h \lesssim \sigma^{1 / 3}, \quad h_{\perp} \lesssim \sqrt{h} \sigma^{-1 / 3} \wedge \sigma^{2 / 3} \wedge \varepsilon^{\nu}
$$

In particular, it is possible to take $h$ of order $\sigma^{1 / 3}$ and $h_{\perp}$ of order $\sigma^{2 / 3} \wedge \varepsilon^{\nu}$. This yields

$$
\mathbb{P}\left\{\phi_{0}(t)>-d_{0} \forall t \in\left[-c_{1} \sigma^{2 / 3},-c_{2} \sigma^{2 / 3}\right]\right\} \leqslant \frac{3}{2} \exp \left\{-\kappa^{\prime} \frac{\sigma^{4 / 3}}{\varepsilon \log \left(\sigma^{-1}\right)}\right\}+\mathcal{O}\left(\frac{\sigma^{4 / 3}}{\varepsilon^{2}} \mathrm{e}^{-\kappa^{\prime} / \sigma^{4 / 3}}\right) .
$$

To summarise, we have thus obtained that with a probability exponentially close to 1 , the transverse component $\phi_{\perp}$ of the solution remains small in $H^{1}$ norm, while the spatial mean $\phi_{0}$ behaves in the same way as the solution of the one-dimensional SDE studied in [5]. In particular, there exist a weak-noise regime in which transitions between stable equilibria are very unlikely, cf. (2.12), and a strong-noise regime, in which transitions are very likely, see (2.13).

An interesting question that remains open so far, is what can be said on regimes where the periodic forcing has a smaller amplitude, so that one stays in the weak-noise regime, but transitions still become likely over very long time spans. In the one-dimensional case, very precise results on the distribution of transition times have been obtained, for instance, in [9, 3]. Generalising these results to the infinite-dimensional situation would require a good understanding of the effect of the dynamics of $\phi_{\perp}$ on transition times. 


\section{Proofs: the stable case}

\subsection{Deterministic case}

In this subsection, we give the proof of Proposition 2.3 on the deterministic dynamics near a stable equilibrium branch $\phi^{*}(t) e_{0}$. We thus consider the deterministic equation

$$
\varepsilon \partial_{t} \phi(t, x)=\Delta \phi(t, x)+f(t, \phi(t, x)),
$$

where $t \in I=[0, T]$ and $f$ satisfies Assumptions 2.1 and 2.2. We are interested in the deviation from the equilibrium branch, given by the difference $\psi(t, \cdot)=\phi(t, \cdot)-\phi^{*}(t) e_{0}$. Using Taylor's formula to expand $f\left(t, \phi^{*}(t) e_{0}+\psi\right)$, we obtain that $\psi$ satisfies the equation

$$
\varepsilon \partial_{t} \psi(t, x)=\Delta \psi(t, x)+a(t) \psi(t, x)+b(t, \psi(t, x))-\varepsilon \frac{\mathrm{d}}{\mathrm{d} t} \phi^{*}(t) e_{0}(x),
$$

where

$$
\begin{aligned}
a(t) & =\partial_{\phi} f\left(t, \phi^{*}(t) e_{0}\right), \\
b(t, \psi) & =\frac{1}{2} \partial_{\phi}^{2} f\left(t, \phi^{*}(t)+\theta \psi\right) \psi^{2} \quad \text { for some } \theta \in[0,1] .
\end{aligned}
$$

This shows in particular that there exist constants $d, M>0$ such that

$$
|b(t, \psi)| \leqslant M \psi^{2}, \quad\left|\partial_{\psi} b(t, \psi)\right| \leqslant M|\psi|
$$

for all $t \in I$ and all $\psi \in \mathbb{R}$ such that $|\psi|<d$.

Proof of Proposition 2.3. Following the main idea of the proof in [24] in the finite-dimensional case, we define a Lyapunov function

$$
V(\psi)=\frac{1}{2}\|\psi\|_{H^{1}}^{2}=\frac{1}{2}\|\psi\|_{L^{2}}^{2}+\frac{L^{2}}{2 \pi^{2}}\|\nabla \psi\|_{L^{2}}^{2} .
$$

Let $\langle\cdot, \cdot\rangle$ denote the $L^{2}$ inner product. Observing that $\|\nabla \psi\|_{L^{2}}^{2}=\langle\nabla \psi, \nabla \psi\rangle=-\langle\psi, \Delta \psi\rangle$, and using self-adjointness of the Laplacian, we obtain that the time derivative of the Lyapunov function along a solution of (3.1) satisfies

$$
\begin{aligned}
\varepsilon \frac{\mathrm{d}}{\mathrm{d} t} V(\psi(t, \cdot))= & \left\langle\psi, \varepsilon \partial_{t} \psi\right\rangle-\frac{L^{2}}{\pi^{2}}\left\langle\Delta \psi, \varepsilon \partial_{t} \psi\right\rangle \\
= & \langle\psi, \Delta \psi\rangle+a(t)\|\psi\|_{L^{2}}^{2}+\langle\psi, b(t, \psi)\rangle-\varepsilon \frac{\mathrm{d}}{\mathrm{d} t} \phi^{*}(t)\left\langle\psi, e_{0}\right\rangle \\
& -\frac{L^{2}}{\pi^{2}}\left[\|\Delta \psi\|_{L^{2}}^{2}+a(t)\langle\Delta \psi, \psi\rangle+\langle\Delta \psi, b(t, \psi)\rangle\right] .
\end{aligned}
$$

In the last line, we have used the fact that $\left\langle\Delta \psi, e_{0}\right\rangle=0$ (here and below, we sometimes write $\psi$ instead of $\psi(t, \cdot)$ in order not to overload the notation). Regrouping terms, and bounding some obviously negative terms above by zero, we get

$$
\varepsilon \frac{\mathrm{d}}{\mathrm{d} t} V(\psi) \leqslant 2 a(t) V(\psi)+\langle\psi, b(t, \psi)\rangle-\frac{L^{2}}{\pi^{2}}\langle\Delta \psi, b(t, \psi)\rangle-\varepsilon \frac{\mathrm{d}}{\mathrm{d} t} \phi^{*}(t)\left\langle\psi, e_{0}\right\rangle .
$$

Let $C_{0}>0$ be a constant to be fixed below. Assume that $\|\psi(0, \cdot)\|_{H^{1}}<C_{0}$, and define the first-exit time

$$
\bar{\tau}=\inf \left\{t>0:\|\psi(t, \cdot)\|_{H^{1}} \geqslant C_{0}\right\} .
$$


By convention, we set $\bar{\tau}=\infty$ whenever $\|\psi(t, \cdot)\|_{H^{1}}<C_{0}$ for all $t \in I$. Thus, for all $t \leqslant \bar{\tau}$ in $I$, we have $\|\psi(t, \cdot)\|_{H^{1}}<C_{0}$. By Sobolev's inequality, this implies that for these $t$, one has

$$
|\psi(t, x)| \leqslant\|\psi(t, \cdot)\|_{L^{\infty}} \leqslant C_{\mathrm{Sob}}\|\psi(t, \cdot)\|_{H^{1}} \leqslant C_{\mathrm{Sob}} C_{0}
$$

for all $x \in \mathbb{R}$ and some numerical constant $C_{\text {Sob }}$. By (3.2), provided $C_{\text {Sob }} C_{0} \leqslant d$, it follows that

$$
|b(t, \psi(t, x))| \leqslant C_{\text {Sob }} M\|\psi(t, \cdot)\|_{H^{1}}^{2},
$$

and thus

$$
\|b(t, \psi(t, \cdot))\|_{L^{2}}^{2} \leqslant C_{\mathrm{Sob}}^{2} M^{2} L\|\psi(t, \cdot)\|_{H^{1}}^{4} .
$$

By the Cauchy-Schwarz inequality, we get

$$
|\langle\psi(t, \cdot), b(t, \psi(t, \cdot))\rangle| \leqslant\|\psi(t, \cdot)\|_{L^{2}}\|b(t, \psi(t, \cdot))\|_{L^{2}} \leqslant C_{\mathrm{Sob}} M L^{1 / 2}\|\psi(t, \cdot)\|_{H^{1}}^{3} .
$$

Furthermore, integration by parts and (3.2) yield

$$
\begin{aligned}
|\langle\Delta \psi(t, \cdot), b(t, \psi(t, \cdot))\rangle| & =\left|\int_{0}^{L} \nabla \psi(t, x)^{2} \partial_{\psi} b(t, \psi(t, x)) \mathrm{d} x\right| \\
& \leqslant M\|\psi(t, \cdot)\|_{L^{\infty}}\|\nabla \psi(t, \cdot)\|_{L^{2}}^{2} \\
& \leqslant C_{\text {Sob }} M\|\psi(t, \cdot)\|_{H^{1}}^{3} .
\end{aligned}
$$

Finally, owing to the implicit function theorem and Assumption 2.2, the derivative of $\phi^{*}(t)$ is bounded by a constant $c$, so that

$$
\left|\frac{\mathrm{d}}{\mathrm{d} t} \phi^{*}(t)\left\langle\psi(t, \cdot), e_{0}\right\rangle\right| \leqslant c\|\psi(t, \cdot)\|_{L^{2}} \leqslant c\|\psi(t, \cdot)\|_{H^{1}} .
$$

Plugging the last three estimates in (3.3), since $a(t)$ is negative and bounded away from zero by Assumption 2.2, we obtain that $V(t)=V(\psi(t, \cdot))$ satisfies

$$
\begin{aligned}
\varepsilon \dot{V} & \leqslant-C_{1} V+C_{2} V^{3 / 2}+\varepsilon C_{3} V^{1 / 2} \\
& \leqslant-C_{1}\left[1-\frac{C_{0}^{1 / 2} C_{2}}{C_{1}}\right] V+\varepsilon C_{3} V^{1 / 2}
\end{aligned}
$$

for all $t \leqslant \bar{\tau}$, and some constants $C_{1}, C_{2}, C_{3}>0$. Choosing $C_{0}$ such that $C_{0}^{1 / 2} \leqslant \frac{C_{1}}{2 C_{2}}$, we obtain

$$
\varepsilon \dot{V} \leqslant-\frac{1}{2} C_{1} V+\varepsilon C_{3} V^{1 / 2}
$$

for all $t \leqslant \bar{\tau}$. Setting $V(t)=Z(t)^{2}$ and dividing by $2 Z(t)$, we get

$$
\varepsilon \dot{Z} \leqslant-\frac{1}{4} C_{1} Z+\frac{1}{2} C_{3} \varepsilon .
$$

Since the variable $W=Z-\frac{2 C_{3}}{C_{1}} \varepsilon$ satisfies $\varepsilon \dot{W} \leqslant-\frac{1}{4} C_{1} W$, Gronwall's inequality yields

$$
W(t) \leqslant W(0) \mathrm{e}^{-C_{1} t /(4 \varepsilon)}
$$

for all $t \leqslant \bar{\tau}$. Thus for any $W(0)$ of order $\varepsilon$, we find that $Z(t)$ remains of order $\varepsilon$ for all $t<\bar{\tau}$, and thus $V(t)$ remains of order $\varepsilon^{2}$. Choosing $\varepsilon_{0}$ small enough and $0<\varepsilon<\varepsilon_{0}$, we obtain in particular that $V(t)<C_{0}$ for all $t<\bar{\tau}$, so that assuming $\bar{\tau}<T$ would lead to a contradiction. We conclude that $\bar{\tau} \geqslant T$, showing that $V(t)=\mathcal{O}\left(\varepsilon^{2}\right)$ for all $t \in I$, which is the claimed result. 
Remark 3.1. Another choice of Lyapunov function would have been

$$
V(t, \psi)=\|\psi\|_{H^{1}}^{2}+\int_{0}^{L} U_{1}(t, \psi(x)) \mathrm{d} x,
$$

where $U_{1}(t, \psi)=U\left(t, \phi^{*}(t) e_{0}+\psi\right)$ is a shifted version of the potential $U$ introduced in (2.2). This function is useful to control the behaviour of solutions of large $H^{1}$-norm. Indeed, one can show that there exist constants $M_{1}, M_{2}>0$ such that

$$
-M_{1} \leqslant V(t, \psi) \leqslant M_{2}\left(1+\|\psi\|_{H^{1}}^{2 p_{0}}\right) \quad \forall t \in I
$$

for all $t \in I$, and that $V(t, \psi(t, \cdot))$ is decreasing at least exponentially fast when it is large.

\subsection{Stochastic case}

We turn now to the analysis of the stochastic equation (2.1) with $\sigma>0$. The equation for the deviation $\psi(t, x)=\phi(t, x)-\bar{\phi}(t, x)$ of the solution from the deterministic solution tracking the stable equilibrium branch $\phi^{*}(t) e_{0}$ reads

$$
\mathrm{d} \psi(t, x)=\frac{1}{\varepsilon}[\Delta \psi(t, x)+\bar{a}(t) \psi(t, x)+b(t, \psi(t, x))] \mathrm{d} t+\frac{\sigma}{\sqrt{\varepsilon}} \mathrm{d} W(t, x),
$$

where

$$
\bar{a}(t)=\partial_{\phi} f(t, \bar{\phi}(t, x))
$$

and $b(t, \psi)$ denotes again a nonlinear term, satisfying bounds analogous to (3.2). We will start by analysing the linear case where $b$ vanishes in Subsection 3.2.1, before turning to the general nonlinear case in Subsection 3.2.2.

\subsubsection{Linear case}

We consider first the linear version of (3.5) given by

$$
\mathrm{d} \psi(t, x)=\frac{1}{\varepsilon}[\Delta \psi(t, x)+\bar{a}(t) \psi(t, x)] \mathrm{d} t+\frac{\sigma}{\sqrt{\varepsilon}} \mathrm{d} W(t, x) .
$$

Denote the eigenvalues of $-\Delta$ by

$$
\mu_{k}=\frac{k^{2} \pi^{2}}{L^{2}}, \quad k \in \mathbb{Z} .
$$

Projecting (3.6) on the $k$ th basis vector $e_{k}$, we obtain

$$
\mathrm{d} \psi_{k}(t)=\frac{1}{\varepsilon} \bar{a}_{k}(t) \psi_{k}(t) \mathrm{d} t+\frac{\sigma}{\sqrt{\varepsilon}} \mathrm{d} W_{k}(t),
$$

where $\bar{a}_{k}(t)=-\mu_{k}+\bar{a}(t)$ and the $\left\{W_{k}(t)\right\}_{t \geqslant 0}$ are independent Wiener processes (see for instance [20]). The solution of (3.8) is a Gaussian process and can be represented by the Ito integral (cf. Duhamel's principle)

$$
\psi_{k}(t)=\frac{\sigma}{\sqrt{\varepsilon}} \int_{0}^{t} \mathrm{e}^{\bar{\alpha}_{k}\left(t, t_{1}\right) / \varepsilon} \mathrm{d} W_{k}\left(t_{1}\right),
$$


where $\bar{\alpha}_{k}\left(t, t_{1}\right)=\int_{t_{1}}^{t} \bar{a}_{k}\left(t_{2}\right) \mathrm{d} t_{2}$. Thus, for each time $t, \psi_{k}(t)$ is characterised by its mean being zero and its variance given by

$$
\operatorname{Var}\left\{\psi_{k}(t)\right\}=\frac{\sigma^{2}}{\varepsilon} \int_{0}^{t} \mathrm{e}^{2 \bar{\alpha}_{k}\left(t, t_{1}\right) / \varepsilon} \mathrm{d} t_{1} .
$$

We may further assume that there are positive constants $\bar{a}_{ \pm}$and $c_{0}^{ \pm}$such that for all $t \in I$

$$
\begin{gathered}
-\bar{a}_{+} \leqslant \bar{a}(t) \leqslant-\bar{a}_{-}, \\
c_{0}^{-}\langle k\rangle^{2} \leqslant \mu_{k}+\bar{a}_{-} \leqslant\left|\bar{a}_{k}(t)\right| \leqslant \mu_{k}+\bar{a}_{+} \leqslant c_{0}^{+}\langle k\rangle^{2},
\end{gathered}
$$

and due to the implicit function theorem we also have the existence of a constant $C$ such that

$$
\left|\bar{a}(t)^{\prime}\right| \leqslant C \quad \forall t \in I=[0, T] .
$$

Lemma 3.2 (Bound on the variance). There exists a constant $C_{0}>0$ such that the variance satisfies the bound

$$
\operatorname{Var}\left\{\psi_{k}(t)\right\} \leqslant C_{0} \frac{\sigma^{2}}{\langle k\rangle^{2}} \quad \forall t \in I .
$$

Proof: Using integration by parts, we obtain

$$
\begin{aligned}
\frac{\operatorname{Var}\left\{\psi_{k}(t)\right\}}{\sigma^{2}} & =\int_{0}^{t} \frac{1}{2 \bar{a}_{k}(s)} \frac{2 \bar{a}_{k}(s)}{\varepsilon} \mathrm{e}^{2 \bar{\alpha}_{k}(t, s) / \varepsilon} \mathrm{d} s \\
& =\frac{1}{2 \bar{a}_{k}(t)}-\frac{1}{2 \bar{a}_{k}(0)} \mathrm{e}^{2 \bar{\alpha}_{k}(t) / \varepsilon}+\frac{1}{2} \int_{0}^{t} \frac{\bar{a}_{k}^{\prime}(s)}{\bar{a}_{k}(s)^{2}} \mathrm{e}^{2 \bar{\alpha}_{k}(t, s) / \varepsilon} \mathrm{d} s,
\end{aligned}
$$

where we write $\bar{\alpha}_{k}(t, 0)=\bar{\alpha}_{k}(t)$ for brevity. The absolute value of integral can be bounded by

$$
\int_{0}^{t} \frac{\left|\bar{a}_{k}^{\prime}(s)\right|}{\left(-\mu_{k}-\bar{a}_{-}\right)^{2}} \mathrm{e}^{-2\left(\mu_{k}+\bar{a}_{-}\right)(t-s) / \varepsilon} \mathrm{d} s \leqslant \frac{C}{2\left(\mu_{k}+\bar{a}_{-}\right)^{3}} \varepsilon .
$$

Therefore,

$$
\frac{\operatorname{Var}\left\{\psi_{k}(t)\right\}}{\sigma^{2}} \leqslant \frac{1+\mathcal{O}(\varepsilon)}{2\left(\mu_{k}+\bar{a}_{-}\right)} \leqslant C_{0} \frac{1}{\langle k\rangle^{2}}
$$

as claimed.

Since each $\psi_{k}(t)$ is a one-dimensional process, we can easily adapt Theorem 2.4 in [4] to obtain the following estimate.

Lemma 3.3. Fix $\gamma>0$. Then for any $k \in \mathbb{Z}$, we have the bound

$$
\mathbb{P}\left\{\sup _{t \in I}\left|\psi_{k}(t)\right| \geqslant h\right\} \leqslant C_{k}(T, \varepsilon) \exp \left\{-\kappa\langle k\rangle^{2} \frac{h^{2}}{\sigma^{2}}\right\},
$$

where $C_{k}(T, \varepsilon)=\frac{2 c_{0}^{-}\langle k\rangle^{2}}{\gamma \varepsilon} T$ and $\kappa=\frac{\mathrm{e}^{-2 \gamma}}{2 C_{0}}$.

Proof: As in [4, Theorem 2.4], we introduce a partition $0=u_{0}<u_{1}<\ldots<u_{N}=T$ of $[0, T]$ by requiring $\bar{\alpha}_{k}\left(u_{l+1}, u_{l}\right)=-\gamma \varepsilon$ for $1 \leqslant l \leqslant N=\left\lfloor c_{0}^{-}\langle k\rangle^{2} T /(\gamma \varepsilon)\right\rfloor$. The proof then follows by approximating the process by a martingale on each interval $\left[u_{l}, u_{l+1}\right]$ and using a Bernstein-type inequality that follows directly from Doob's submartingale inequality. 
Proof of Theorem 2.4 in the linear case. Fix constants $\eta, \rho>0$ and $s \in\left(0, \frac{1}{2}\right)$ such that $s=\frac{1}{2}-\rho$. For every decomposition $h^{2}=\sum_{k \in \mathbb{Z}} h_{k}^{2}$ one has

$$
\begin{aligned}
\mathbb{P}\left\{\tau_{\mathcal{B}(h)}<t\right\} & =\mathbb{P}\left\{\sup _{t \in I}\|\psi(t, \cdot)\|_{H^{s}}^{2} \geqslant h^{2}\right\} \\
& =\mathbb{P}\left\{\sup _{t \in I} \sum_{k \in \mathbb{Z}}\langle k\rangle^{2 s}\left|\psi_{k}(t)\right|^{2} \geqslant h^{2}\right\} \\
& \leqslant \sum_{k \in \mathbb{Z}} \mathbb{P}\left\{\sup _{t \in I}\left|\psi_{k}(t)\right|^{2} \geqslant h_{k}^{2}\langle k\rangle^{-2 s}\right\} \\
& \leqslant \sum_{k \in \mathbb{Z}} C_{k}(T, \varepsilon) \exp \left\{-\kappa \frac{h_{k}^{2}}{\sigma^{2}}\langle k\rangle^{2-2 s}\right\} .
\end{aligned}
$$

Choosing

$$
h_{k}^{2}=C(\eta, s) h^{2}\langle k\rangle^{-2+2 s+\eta},
$$

the condition $h^{2}=\sum_{k \in \mathbb{Z}} h_{k}^{2}$ yields

$$
C(\eta, s)=\frac{1}{\sum_{k \in \mathbb{Z}}\langle k\rangle^{-2+2 s+\eta}} .
$$

Since the Riemann zeta function $\zeta(v)=\sum_{n \geqslant 1} n^{-v}$ converges for $v>1$, we get

$$
\sum_{k \in \mathbb{Z}} \frac{1}{\langle k\rangle^{2-2 s-\eta}} \leqslant 1+2 \sum_{k=1}^{\infty} \frac{1}{k^{2-2 s-\eta}}=1+2 \zeta(2-2 s-\eta)<\infty \quad \forall 0<\eta<2 \rho .
$$

With $h_{k}$ given by (3.9) and $\eta$ satisfying this condition, we get

$$
\begin{aligned}
\mathbb{P}\left\{\sup _{t \in I}\|\psi(t, \cdot)\|_{H^{s}}^{2} \geqslant h^{2}\right\} & \leqslant \sum_{k \in \mathbb{Z}} C_{k}(T, \varepsilon) \exp \left\{-\kappa C(\eta, s) \frac{h^{2}}{\sigma^{2}}\langle k\rangle^{\eta}\right\} \\
& =\alpha_{T} \sum_{k \in \mathbb{Z}}\langle k\rangle^{2} \mathrm{e}^{-\beta\langle k\rangle^{\eta}}
\end{aligned}
$$

where we write $\alpha_{T}=\frac{2 c_{0}^{-}}{\gamma \varepsilon} T$ and $\beta=\kappa C(\eta, s) \frac{h^{2}}{\sigma^{2}}$ for simplicity. In order to bound the sum, we write

$$
f(x)=\left(1+x^{2}\right) \mathrm{e}^{-\beta\left(1+x^{2}\right)^{\eta / 2}} .
$$

Note that we may assume that $f$ is decreasing by taking $h / \sigma$ larger than an $\eta$-dependent constant of order 1 (which we may do, because otherwise the result is trivially true). Therefore, we obtain

$$
\sum_{k \in \mathbb{Z}} f(k)=f(0)+2 \sum_{k=1}^{\infty} f(k) \leqslant \mathrm{e}^{-\beta}+2 \int_{0}^{\infty} f(x) \mathrm{d} x .
$$

In what follows, we show that the integral

$$
I=\int_{0}^{\infty} f(x) \mathrm{d} x=\int_{0}^{\infty}\left(1+x^{2}\right) \mathrm{e}^{-\beta\left(1+x^{2}\right)^{\eta / 2}} \mathrm{~d} x
$$


is finite, and, more precisely, has order $\beta^{-1 / 2} \mathrm{e}^{-\beta}$. We first make the change of variable $y=$ $\beta\left(1+x^{2}\right)^{\eta / 2}$, yielding

$$
I=\frac{1}{\eta \beta^{4 / \eta}} \int_{\beta}^{\infty} \mathrm{e}^{-y} \frac{y^{4 / \eta-1}}{\sqrt{\left(\frac{y}{\beta}\right)^{2 / \eta}-1}} \mathrm{~d} y .
$$

The further change of variable $y=\beta+z$ gives

$$
I=\frac{\mathrm{e}^{-\beta}}{\eta \beta^{4 / \eta}} \int_{0}^{\infty} \mathrm{e}^{-z} \frac{(\beta+z)^{4 / \eta-1}}{\sqrt{\left(1+\frac{z}{\beta}\right)^{2 / \eta}-1}} \mathrm{~d} z .
$$

Using Taylor's formula, we get the lower bound

$$
\left(1+\frac{z}{\beta}\right)^{2 / \eta}-1 \geqslant \frac{2}{\eta} \frac{z}{\beta}
$$

Therefore,

$$
\begin{aligned}
I & \leqslant \frac{\mathrm{e}^{-\beta}}{\eta \beta^{4 / \eta}} \sqrt{\frac{\beta \eta}{2}} \int_{0}^{\infty} \frac{\mathrm{e}^{-z}}{\sqrt{z}}(\beta+z)^{4 / \eta-1} \mathrm{~d} z \\
& =\frac{\mathrm{e}^{-\beta}}{\eta \beta^{4 / \eta}} \sqrt{\frac{\beta \eta}{2}}\left[\int_{0}^{\beta} \frac{\mathrm{e}^{-z}}{\sqrt{z}}(\beta+z)^{4 / \eta-1} \mathrm{~d} z+\int_{\beta}^{\infty} \frac{\mathrm{e}^{-z}}{\sqrt{z}}(\beta+z)^{4 / \eta-1} \mathrm{~d} z\right] \\
& \leqslant \frac{\mathrm{e}^{-\beta}}{\sqrt{2 \eta} \beta^{4 / \eta-1 / 2}}(2 \beta)^{4 / \eta-1} \int_{0}^{\beta} \frac{\mathrm{e}^{-z}}{\sqrt{z}} \mathrm{~d} z+\frac{\mathrm{e}^{-\beta}}{\sqrt{2 \eta} \beta^{4 / \eta-1 / 2}} 2^{4 / \eta-1} \int_{\beta}^{\infty} \frac{\mathrm{e}^{-z}}{\sqrt{z}} z^{4 / \eta-1} \mathrm{~d} z \\
& \leqslant c_{1}(\eta) \frac{\mathrm{e}^{-\beta}}{\sqrt{\beta}}+c_{2}(\eta) \frac{\mathrm{e}^{-\beta}}{\beta^{4 / \eta-1 / 2}},
\end{aligned}
$$

where $c_{1}(\eta)$ and $c_{2}(\eta)$ are bounded uniformly in $\beta$, provided $\eta<8$. It follows that

$$
\begin{aligned}
\sum_{k \in \mathbb{Z}}\langle k\rangle^{2} \mathrm{e}^{-\beta\langle k\rangle^{\eta}} & \leqslant \mathrm{e}^{-\beta}+2 c_{1}(\eta) \frac{\mathrm{e}^{-\beta}}{\sqrt{\beta}}+2 c_{2}(\eta) \frac{\mathrm{e}^{-\beta}}{\beta^{4 / \eta-1 / 2}} \\
& =\mathrm{e}^{-\kappa C(\eta, s) h^{2} / \sigma^{2}}\left[1+\bar{c}_{1}(\eta) \frac{\sigma}{h}+\bar{c}_{2}(\eta)\left(\frac{\sigma^{2}}{h^{2}}\right)^{4 / \eta-1 / 2}\right] .
\end{aligned}
$$

We thus conclude that

$$
\begin{aligned}
\mathbb{P}\left\{\sup _{t \in I}\|\psi(t, \cdot)\|_{H^{s}}^{2} \geqslant h^{2}\right\} & \leqslant \alpha_{T}\left[1+\bar{c}_{1}(\eta) \frac{\sigma}{h}+\bar{c}_{2}(\eta)\left(\frac{\sigma^{2}}{h^{2}}\right)^{4 / \eta-1 / 2}\right] \mathrm{e}^{-\kappa C(\eta, s) h^{2} / \sigma^{2}} \\
& =: C(\gamma, T, \varepsilon, s) \mathrm{e}^{-\kappa C(\eta, s) h^{2} / \sigma^{2}}
\end{aligned}
$$

where we can fix, for instance, $\eta=\rho=\frac{1}{2}-s$, which yields $C(\eta, s)=\left[1+2 \zeta\left(\frac{3}{2}-s\right)\right]^{-1}$ by (3.10).

\subsubsection{Nonlinear case}

We return now to the study of the general nonlinear equation (3.5). By Duhamel's principle, its solution satisfies the equation

$$
\begin{aligned}
\psi(t, \cdot) & =\frac{\sigma}{\sqrt{\varepsilon}} \int_{0}^{t} \mathrm{e}^{\bar{\alpha}\left(t, t_{1}\right) / \varepsilon} \mathrm{e}^{\left[\left(t-t_{1}\right) / \varepsilon\right] \Delta} \mathrm{d} W\left(t_{1}, \cdot\right)+\frac{1}{\varepsilon} \int_{0}^{t} \mathrm{e}^{\bar{\alpha}\left(t, t_{1}\right) / \varepsilon} \mathrm{e}^{\left[\left(t-t_{1}\right) / \varepsilon\right] \Delta} b\left(t_{1}, \psi\left(t_{1}, \cdot\right)\right) \mathrm{d} t_{1} \\
& =\psi^{0}(t, \cdot)+\psi^{1}(t, \cdot)
\end{aligned}
$$


Here $\bar{\alpha}\left(t, t_{1}\right)=\int_{t_{1}}^{t} \bar{a}(u) \mathrm{d} u$, and $\mathrm{e}^{t \Delta}$ denotes the heat kernel. We notice that $\psi^{0}(t, x)$ is the solution of the linear equation (3.6), and therefore satisfies the estimate (3.11).

In what follows, we give some technical results that will be needed several times in order to show that $\psi^{1}(t, \cdot)$ belongs to a certain Sobolev space included in $H^{s}$.

Lemma 3.4. Let the potential $U(t, \phi)$ satisfy Assumption 2.1 and assume $\psi(t, \cdot) \in H^{s}$ for all $0<s<\frac{1}{2}$. Then

$$
\beta(t)=b(t, \psi(t, \cdot))
$$

belongs to $H^{r}$ for all $r<\frac{1}{2}$. Furthermore, for all $r<\frac{1}{2}-\left(2 p_{0}+1\right)\left(\frac{1}{2}-s\right)$, there exists $C(r, s)<\infty$ such that

$$
\|\beta(t)\|_{H^{r}} \leqslant C(r, s) \max \left\{\|\psi\|_{H^{s}}^{2},\|\psi\|_{H^{s}}^{2 p_{0}+1}\right\} .
$$

Proof: Consider first the case where $U$ is a polynomial in $\psi$ of degree $2 p_{0}$. Then $f(t, \psi)$ and $\beta(t)$ are polynomials of degree $2 p_{0}+1$. Applying Young's inequality (A.1), we obtain by induction that if $\psi(t, \cdot) \in H^{\frac{1}{2}-\kappa}$ for a $\kappa>0$, then for any $k \geqslant 2, \psi(t, \cdot)^{k} \in H^{r}$ for any $r<\frac{1}{2}-k \kappa$. It follows that $\beta(t) \in H^{r}$ for all $r<\frac{1}{2}-\left(2 p_{0}+1\right) \kappa$. Since $\kappa>0$ is arbitrary, we conclude that indeed $\beta(t) \in H^{r}$ for all $r<\frac{1}{2}$. The bound (3.13) is then a consequence of Young's inequality (A.1), the bound (3.2) on $b(t, \psi)$ for small $\psi$, and the fact that $\beta(t)$ is a polynomial of degree $2 p_{0}+1$.

Consider now the general case. By Assumption 2.1, $f(t, \psi)$ and $\beta(t)$ are each the sum of a polynomial of degree $2 p_{0}+1$ and a bounded function $g(t, \psi)$. For $0<s<2$ and $1 \leqslant p, q \leqslant \infty$, consider the Besov space $\mathcal{B}_{p}^{s, q}$. Then, [11] shows that there exists a constant $R(p, q, s, M)>0$ such that for all $\psi$ in the positive cone, $\left(\mathcal{B}_{p}^{s, q}\right)^{+}$, we have

$$
\|g \circ \psi\|_{\mathcal{B}_{p}^{s, q}} \leqslant R(p, q, s, M)\|\psi\|_{\mathcal{B}_{p}^{s, q}} .
$$

In particular, whenever $p=q=2$ the Besov space $\mathcal{B}_{2}^{s, 2}$ is nothing but the Sobolev space $H^{s}$. Thus, if $\psi(t, \cdot) \in H^{s}$, then $g \circ \psi \in H^{s}$ and $\beta(t) \in H^{r}$ for all $r<\frac{1}{2}$.

Lemma 3.5 (Schauder-type estimate). Assume $\beta \in H^{r}$ for some $r \in\left(0, \frac{1}{2}\right)$. Then for all $q<r+2$, there exists a constant $M(q, r)<\infty$ such that

$$
\left\|\mathrm{e}^{t \Delta} \beta\right\|_{H^{q}} \leqslant M(q, r) t^{-\frac{q-r}{2}}\|\beta\|_{H^{r}}
$$

for all $t>0$.

Proof: Let $\gamma=\frac{q-r}{2}$. Writing the Fourier expansion of $\beta$ as $\beta(x)=\sum_{k \in \mathbb{Z}} \beta_{k} e_{k}(x)$, we have

$$
\mathrm{e}^{t \Delta} \beta(x)=\sum_{k \in \mathbb{Z}} \mathrm{e}^{-\mu_{k} t} \beta_{k} e_{k}(x),
$$

where the $-\mu_{k}$ are the eigenvalues of the Laplacian, cf. (3.7). By definition of the fractional Sobolev norm, we obtain

$$
\begin{aligned}
\left\|t^{\gamma} e^{t \Delta} \beta\right\|_{H^{q}}^{2} & =\sum_{k}\langle k\rangle^{2 q} t^{2 \frac{q-r}{2}} \mathrm{e}^{-2 \mu_{k} t} \beta_{k}^{2} \\
& \leqslant \sum_{k}\left[\langle k\rangle^{2} t\right]^{q-r} \mathrm{e}^{-c_{0}^{-}\langle k\rangle^{2} t}\langle k\rangle^{2 r} \beta_{k}^{2} \\
& =\sum_{k} H\left(\langle k\rangle^{2} t\right)\langle k\rangle^{2 r} \beta_{k}^{2}
\end{aligned}
$$


where $H(z)=z^{q-r} \mathrm{e}^{-c_{0}^{-} z}$ reaches its maximum at $z^{*}=\frac{q-r}{c_{0}^{-}}$. Therefore,

$$
0 \leqslant H(z) \leqslant M(q, r)^{2}=H\left(z^{*}\right)=\left(\frac{q-r}{c_{0}^{-}}\right)^{q-r} \mathrm{e}^{-(q-r)}
$$

for all $z \geqslant 0$. We conclude that for all $t \in I$,

$$
\left\|t^{\frac{q-r}{2}} \mathrm{e}^{t \Delta} \beta\right\|_{H^{q}}^{2} \leqslant \sum_{k} M(q, r)^{2}\langle k\rangle^{2 r} \beta_{k}^{2}=M(q, r)^{2}\|\beta\|_{H^{r}}^{2}
$$

as claimed.

Applying this result to the term $\psi^{1}(t, \cdot)$ defined in (3.12), we obtain the following key estimate.

Corollary 3.6. Assume there exists $r \in\left(0, \frac{1}{2}\right)$ such that $\beta(t) \in H^{r}$ for all $t \in I$. Then for all $q<r+2$, there exists a constant $M^{\prime}(q, r)<\infty$ such that for all $t \in I$, one has $\psi^{1}(t, \cdot) \in H^{q}$ and

$$
\left\|\psi^{1}(t, \cdot)\right\|_{H^{q}} \leqslant M^{\prime}(q, r) \varepsilon^{\frac{q-r}{2}-1} \sup _{0 \leqslant t_{1} \leqslant t}\left\|\beta\left(t_{1}\right)\right\|_{H^{r}} .
$$

Proof: Note that $\bar{\alpha}\left(t, t_{1}\right) \leqslant-\frac{c_{0}^{-}}{2}\left(t-t_{1}\right)$ whenever $t_{1} \leqslant t$. Furthermore, the previous result implies that for any $q<r+2$, one has

$$
\left\|\mathrm{e}^{(t / \varepsilon) \Delta} \beta(t)\right\|_{H^{q}} \leqslant M(q, r)\left(\frac{\varepsilon}{t}\right)^{\frac{q-r}{2}}\|\beta(t)\|_{H^{r}} .
$$

Therefore

$$
\begin{aligned}
\left\|\psi^{1}(t, x)\right\|_{H^{q}} & \leqslant \frac{1}{\varepsilon} \int_{0}^{t} \mathrm{e}^{-c_{0}^{-}\left(t-t_{1}\right) /(2 \varepsilon)}\left\|\mathrm{e}^{\left[\left(t-t_{1}\right) / \varepsilon\right] \Delta} \beta\left(t_{1}\right)\right\|_{H^{q}} \mathrm{~d} t_{1} \\
& \leqslant M(q, r) \varepsilon^{\frac{q-r}{2}-1} \sup _{0 \leqslant t_{1} \leqslant t}\left\|\beta\left(t_{1}\right)\right\|_{H^{r}} \int_{0}^{t}\left(t-t_{1}\right)^{-\frac{q-r}{2}} \mathrm{~d} t_{1},
\end{aligned}
$$

and the integral over $t_{1}$ is bounded whenever $q-r<2$.

Now, if $s \leqslant q$ then $H^{q} \subset H^{s}$ and thus $\psi^{1}(t, \cdot) \in H^{s}$ whenever $\psi(t, \cdot) \in H^{s}$. With these results, we can now prove Theorem 2.4 for the nonlinear case.

Proof of Theorem 2.4 For every decomposition $h=h_{0}+h_{1}$ with $h_{0}, h_{1}>0$, one has

$$
\begin{aligned}
\mathbb{P}\left\{\tau_{\mathcal{B}(h)}<t\right\}= & \mathbb{P}\left\{\sup _{0 \leqslant t \leqslant T \wedge \tau_{\mathcal{B}(h)}}\|\psi(t, \cdot)\|_{H^{s}}>h\right\} \\
\leqslant & \mathbb{P}\left\{\sup _{0 \leqslant t \leqslant T \wedge \tau_{\mathcal{B}(h)}}\left\|\psi^{1}(t, \cdot)\right\|_{H^{s}}+\left\|\psi^{0}(t, \cdot)\right\|_{H^{s}}>h\right\} \\
\leqslant & \mathbb{P}\left\{\sup _{0 \leqslant t \leqslant T}\left\|\psi^{0}(t, \cdot)\right\|_{H^{s}}>h_{0}\right\} \\
& +\mathbb{P}\left\{\sup _{0 \leqslant t \leqslant T \wedge \tau_{\mathcal{B}(h)}}\left\|\psi^{1}(t, \cdot)\right\|_{H^{s}}>h_{1}, \sup _{0 \leqslant t \leqslant T}\left\|\psi^{0}(t, \cdot)\right\|_{H^{s}} \leqslant h_{0}\right\} .
\end{aligned}
$$


The first term on the right-hand side can be estimated by (3.11). Furthermore, for all $t<\tau_{\mathcal{B}(h)}$, we have $\|\beta(t)\|_{H^{r}} \leqslant M\|\psi(t, \cdot)\|_{H^{s}}^{2} \leqslant M h^{2}$, so that

$$
\left\|\psi^{1}(t, x)\right\|_{H^{q}} \leqslant M^{\prime}(q, r) \varepsilon^{\frac{q-r}{2}-1} M h^{2} .
$$

Choosing $h_{1}=M^{\prime}(q, r) \varepsilon^{\frac{q-r}{2}-1} M h^{2}$, we get

$$
\mathbb{P}\left\{\sup _{0 \leqslant t \leqslant T \wedge \tau_{\mathcal{B}(h)}}\left\|\psi^{1}(t, x)\right\|_{H^{s}}>h_{1}, \sup _{0 \leqslant t \leqslant T}\left\|\psi^{0}(t, x)\right\|_{H^{s}} \leqslant h_{0}\right\}=0 .
$$

We thus obtain the result by choosing $h_{0}=h-h_{1}=h-M^{\prime}(q, r) \varepsilon^{\frac{q-r}{2}-1} M h^{2}=h\left(1-\mathcal{O}\left(h / \varepsilon^{\nu}\right)\right)$ and $\nu=1-\frac{q-r}{2}$.

\section{Proofs: bifurcations}

Before entering the detailed analysis, we make a preliminary change of variables yielding the form (2.6) of the equations. Let $\alpha, \beta, \gamma \in \mathbb{R}$. Using the scaling $t=\alpha \bar{t}, x=\beta \bar{x}$ and $\phi=\gamma \bar{\phi}$ in (2.4), we obtain the following SPDE. For all $\bar{x} \in\left[0, \bar{L}=\frac{L}{\beta}\right]$, one has

$$
\mathrm{d} \bar{\phi}(\bar{t}, \bar{x})=\frac{1}{\bar{\varepsilon}}\left[\Delta \bar{\phi}(\bar{t}, \bar{x})+\bar{\alpha} g(\bar{t})-\bar{\beta} \bar{\phi}(\bar{t}, \bar{x})^{2}-\bar{\gamma} b(\bar{t}, \bar{\phi}(\bar{t}, \bar{x}))\right] \mathrm{d} \bar{t}+\frac{\bar{\sigma}}{\sqrt{\bar{\varepsilon}}} \mathrm{d} W(\bar{t}, \bar{x}),
$$

where $\bar{\varepsilon}=\frac{\beta^{2}}{\alpha} \varepsilon, \bar{\alpha}=\frac{\alpha^{2} \beta^{2}}{\gamma}, \bar{\beta}=\gamma \beta^{2}, \bar{\gamma}=\gamma^{2} \beta^{2}$ and $\bar{\sigma}=\frac{\sqrt{\beta}}{\alpha \gamma} \sigma$ (below, we drop the bars in order not to overload the notation). We now apply the decomposition (2.5) of the solution in its mean and oscillating part. Taylor's formula yields

$$
b\left(t, \phi_{0} e_{0}+\phi_{\perp}\right)=b\left(t, \phi_{0} e_{0}\right)+\partial_{\phi} b\left(t, \phi_{0} e_{0}\right) \phi_{\perp}+\frac{1}{2} \partial_{\phi}^{2} b\left(t, \phi_{0} e_{0}\right) \phi_{\perp}^{2}+R\left(t, \phi_{0} e_{0}, \phi_{\perp}\right),
$$

where

$$
R\left(t, \phi_{0} e_{0}, \phi_{\perp}\right)=\frac{1}{6} \partial_{\phi}^{3} b\left(t, \phi_{0} e_{0}+\theta \phi_{\perp}\right) \phi_{\perp}^{3}
$$

for some $\theta \in[0,1]$. Therefore, the spatially constant part $\phi_{0}(t)$ of the solution $\phi(t, x)$ satisfies the equation

$$
\begin{aligned}
\mathrm{d} \phi_{0}(t)= & \frac{1}{\varepsilon}\left\langle e_{0}, \Delta \phi(t, \cdot)+\alpha g(t)-\beta \phi(t, \cdot)^{2}-\gamma b(t, \phi(t, \cdot))\right\rangle \mathrm{d} t+\frac{\sigma}{\sqrt{\varepsilon}}\left\langle e_{0}, \mathrm{~d} W(t, \cdot)\right\rangle \\
=\frac{1}{\varepsilon}\left[\alpha \sqrt{L} g(t)-\frac{\beta}{\sqrt{L}} \phi_{0}(t)^{2}-\frac{\beta}{\sqrt{L}}\left\|\phi_{\perp}\right\|_{L^{2}}^{2}-\gamma \sqrt{L} b\left(t, \phi_{0}(t) e_{0}\right)\right. & \left.\quad-\frac{\gamma}{2 \sqrt{L}} \partial_{\phi}^{2} b\left(t, \phi_{0}(t) e_{0}\right)\left\|\phi_{\perp}(t, \cdot)\right\|_{L^{2}}^{2}-\gamma\left\langle e_{0}, R\left(t, \phi_{0}(t) e_{0}, \phi_{\perp}(t, \cdot)\right)\right\rangle\right] \mathrm{d} t \\
& +\frac{\sigma}{\sqrt{\varepsilon}} \mathrm{d} W_{0}(t) .
\end{aligned}
$$


On the other hand, the mean zero part $\phi_{\perp}(t, x)=\phi(t, x)-\phi_{0}(t) e_{0}(x)$ satisfies

$$
\begin{aligned}
\mathrm{d} \phi_{\perp}(t, x)= & \mathrm{d} \phi(t, x)-\mathrm{d} \phi_{0}(t) e_{0}(x) \\
=\frac{1}{\varepsilon}\left[\Delta \phi_{\perp}(t, x)-\left(2 \frac{\beta}{\sqrt{L}} \phi_{0}(t)+\gamma \partial_{\phi} b\left(t, \phi_{0}(t) e_{0}(x)\right)\right) \phi_{\perp}(t, x)\right. & -\left(\beta+\frac{\gamma}{2} \partial_{\phi}^{2} b\left(t, \phi_{0}(t) e_{0}(x)\right)\right)\left(\phi_{\perp}(t, x)^{2}-\frac{1}{L}\left\|\phi_{\perp}(t, \cdot)\right\|_{L^{2}}^{2}\right) \\
& \quad-\gamma\left[R\left(t, \phi_{0}(t) e_{0}(x), \phi_{\perp}(t, x)\right)-\frac{1}{\sqrt{L}}\left\langle e_{0}, R\left(t, \phi_{0}(t) e_{0}, \phi_{\perp}(t, \cdot)\right)\right\rangle\right] \mathrm{d} t \\
+ & \frac{\sigma}{\sqrt{\varepsilon}} \mathrm{d} W_{\perp}(t, x) .
\end{aligned}
$$

Choosing $\alpha=\frac{1}{\sqrt{L}}, \beta=\sqrt{L}$ and $\gamma=\frac{1}{\sqrt{L}}$ yields the coupled SDE-SPDE system (2.6) with

$$
\begin{aligned}
b_{0}\left(t, \phi_{0}, \phi_{\perp}\right)= & -\left(1+\frac{1}{2 L} \partial_{\phi}^{2} b\left(t, \phi_{0} e_{0}\right)\right)\left\|\phi_{\perp}\right\|_{L^{2}}^{2}-\frac{1}{\sqrt{L}}\left\langle e_{0}, R\left(t, \phi_{0} e_{0}, \phi_{\perp}\right)\right\rangle, \\
a\left(t, \phi_{0}\right)= & -2 \phi_{0}-\frac{1}{\sqrt{L}} \partial_{\phi} b\left(t, \phi_{0} e_{0}\right), \\
b_{\perp}\left(t, \phi_{0}, \phi_{\perp}\right)= & -\sqrt{L}\left(1+\frac{1}{2 L} \partial_{\phi}^{2} b\left(t, \phi_{0} e_{0}\right)\right)\left(\phi_{\perp}(\cdot)^{2}-\frac{1}{L}\left\|\phi_{\perp}\right\|_{L^{2}}^{2}\right) \\
& -\frac{1}{\sqrt{L}} R\left(t, \phi_{0} e_{0}, \phi_{\perp}\right)+\frac{1}{L}\left\langle e_{0}, R\left(t, \phi_{0} e_{0}, \phi_{\perp}\right)\right\rangle .
\end{aligned}
$$

Note that $b_{0}$ and $b_{\perp}$ are no longer local non-linearities, since they involve integrals over the whole torus. This remains, however, a relatively harmless non-locality, that will not cause any problems.

We now derive a number of bounds on the remainder terms $b_{0}$ and $b_{\perp}$. By similar arguments as in the proof of Proposition 2.3, there exist constants $d, \bar{d}>0$ such that whenever $\left|\phi_{0}\right|<\bar{d}$ and $\left\|\phi_{\perp}\right\|_{H^{1}}<d$, one has

$$
\left|R\left(t, \phi_{0} e_{0}, \phi_{\perp}(x)\right)\right| \leqslant M\left|\phi_{\perp}(x)\right|^{3} \leqslant M C_{\mathrm{Sob}}\left\|\phi_{\perp}\right\|_{H^{1}}^{3}
$$

for some finite constant $M$. Therefore, under these conditions on $\phi_{0}$ and $\phi_{\perp}$, we obtain

$$
\left|b_{\perp}\left(t, \phi_{0}, \phi_{\perp}(x)\right)\right| \leqslant M_{1}\left\|\phi_{\perp}\right\|_{H^{1}}^{3}
$$

for some constant $M_{1}$. Furthermore, the same argument as in Lemma 3.4 shows that for all $r<\frac{1}{2}-\left(2 p_{0}+1\right)\left(\frac{1}{2}-s\right)$, there exists $C(r, s)<\infty$ such that

$$
\left\|R\left(t, \phi_{0} e_{0}, \phi_{\perp}\right)\right\|_{H^{r}} \leqslant C(r, s) \max \left\{\left\|\phi_{\perp}\right\|_{H^{s}}^{3},\left\|\phi_{\perp}\right\|_{H^{s}}^{2 p_{0}-1}\right\} .
$$

Combining this with the Cauchy-Schwarz inequality, we obtain the existence of a constant $M_{2}$ such that the bounds

$$
\begin{gathered}
\left|b_{0}\left(t, \phi_{0}, \phi_{\perp}\right)\right| \leqslant M_{2} \max \left\{\left\|\phi_{\perp}\right\|_{H^{s}}^{2},\left\|\phi_{\perp}\right\|_{H^{s}}^{2 p_{0}-1}\right\}, \\
\left\|b_{\perp}\left(t, \phi_{0}, \phi_{\perp}\right)\right\|_{H^{r}}
\end{gathered}
$$

hold for all $\phi_{0} \in \mathbb{R}$ such that $\left|\phi_{0}\right|<\bar{d}$. 


\subsection{Deterministic case}

We start by investigating the deterministic behaviour of the solution $\left(\phi_{0}(t), \phi_{\perp}(t, \cdot)\right)$. The deterministic equation for $\phi_{\perp}(t, x)$ is given by

$$
\mathrm{d} \phi_{\perp}(t, x)=\frac{1}{\varepsilon}\left[\Delta \phi_{\perp}(t, x)+a\left(t, \phi_{0}(t)\right) \phi_{\perp}(t, x)+b_{\perp}\left(t, \phi_{0}(t), \phi_{\perp}(t, x)\right)\right] \mathrm{d} t .
$$

Proof of Proposition 2.8. The proof is almost the same as the proof of Proposition 2.3, so that we only comment on the differences. Here we define the Lyapunov function

$$
V\left(\phi_{\perp}\right)=\frac{1}{2}\left\|\phi_{\perp}\right\|_{H^{1}}^{2}=\frac{1}{2}\left\|\phi_{\perp}\right\|_{L^{2}}^{2}+\frac{L^{2}}{2 \pi^{2}}\left\|\nabla \phi_{\perp}\right\|_{L^{2}}^{2} .
$$

Its time derivative satisfies

$$
\begin{aligned}
& \varepsilon \frac{\mathrm{d}}{\mathrm{d} t} V\left(\phi_{\perp}(t, \cdot)\right)=\left\langle\phi_{\perp}, \Delta \phi_{\perp}\right\rangle+a\left(t, \phi_{0}\right)\left\|\phi_{\perp}\right\|_{L^{2}}^{2}+\left\langle\phi_{\perp}, b_{\perp}\left(t, \phi_{0}, \phi_{\perp}\right)\right\rangle \\
&-\frac{L^{2}}{\pi^{2}}\left[\left\|\Delta \phi_{\perp}\right\|_{L^{2}}^{2}+a\left(t, \phi_{0}\right)\left\langle\Delta \phi_{\perp}, \phi_{\perp}\right\rangle+\left\langle\Delta \phi_{\perp}, b_{\perp}\left(t, \phi_{0}, \phi_{\perp}\right)\right\rangle\right] \\
& \leqslant 2 a\left(t, \phi_{0}\right) V\left(\phi_{\perp}\right)+\left\langle\phi_{\perp}, b_{\perp}\left(t, \phi_{0}, \phi_{\perp}\right)\right\rangle-\frac{L^{2}}{\pi^{2}}\left\langle\Delta \phi_{\perp}, b_{\perp}\left(t, \phi_{0}, \phi_{\perp}\right)\right\rangle .
\end{aligned}
$$

Using (4.2) and the Cauchy-Schwarz inequality, we obtain that for $\phi_{0}$ and $\left\|\phi_{\perp}\right\|_{H^{1}}$ small enough, the term $\left\langle\phi_{\perp}, b_{\perp}\left(t, \phi_{0}, \phi_{\perp}\right)\right\rangle$ has order $\left\|\phi_{\perp}\right\|_{H^{1}}^{3}$. As for the last term, it follows from the expression (4.1) of $b_{\perp}$ that it has the form

$$
\begin{aligned}
\left\langle\Delta \phi_{\perp}, b_{\perp}\left(t, \phi_{0}, \phi_{\perp}\right)\right\rangle= & A(t)\left\langle\Delta \phi_{\perp}, \phi_{\perp}^{2}\right\rangle+B(t)\left\langle\Delta \phi_{\perp}, 1\right\rangle\left\|\phi_{\perp}\right\|_{L^{2}}^{2} \\
& -\frac{1}{\sqrt{L}}\left\langle\Delta \phi_{\perp}, R\left(t, \phi_{0} e_{0}, \phi_{\perp}\right)\right\rangle+\frac{1}{L}\left\langle\Delta \phi_{\perp}, 1\right\rangle\left\langle e_{0}, R\left(t, \phi_{0} e_{0}, \phi_{\perp}\right)\right\rangle
\end{aligned}
$$

for some bounded functions $A$ and $B$. The first term on the right-hand side can be bounded using integration by parts. The third one has order $\left\|\phi_{\perp}\right\|_{H^{1}}^{2}\left\|\phi_{\perp}\right\|_{L^{\infty}}^{2}$, and the other two terms vanish because $\left\langle\Delta \phi_{\perp}, 1\right\rangle=0$. It follows that $\left\langle\Delta \phi_{\perp}, b_{\perp}\left(t, \phi_{0}, \phi_{\perp}\right)\right\rangle$ has also order $\left\|\phi_{\perp}\right\|_{H^{1}}^{3}$, provided $\phi_{0}$ and $\left\|\phi_{\perp}\right\|_{H^{1}}$ are small enough.

Writing as before $\bar{\tau}$ for the first-exit time from the set $\left\{V\left(\phi_{\perp}(t, \cdot)\right) \leqslant C_{0}\right\}$, we obtain

$$
\varepsilon \dot{V} \leqslant-C_{1} V+C_{2} V^{3 / 2} \leqslant-C_{1}\left[1-\frac{C_{0}^{1 / 2} C_{2}}{C_{1}}\right] V
$$

for all $t \leqslant \bar{\tau}$, and some constants $C_{1}, C_{2}>0$. Choosing $C_{0}$ such that $C_{0}^{1 / 2} \leqslant \frac{C_{1}}{2 C_{2}}$, we obtain

$$
\varepsilon \dot{V} \leqslant-\frac{1}{2} C_{1} V,
$$

which allows to show that there exists a particular solution satisfying $V(t)=0$ for all $t \in I$. As for $\phi_{0}(t)$, it obeys the ODE

$$
\varepsilon \dot{\phi}_{0}(t)=g(t)-\phi_{0}(t)^{2}-b\left(t, \phi_{0}(t) e_{0}\right),
$$

which can be analysed in exactly the same way as in [5], concluding the proof. 


\subsection{Stochastic case}

We consider now the coupled SDE-SPDE system (2.6) with $\sigma>0$. We start by analysing the dynamics of $\phi_{\perp}(t, x)$ for a given realisation of $\phi_{0}(t)$. The SPDE

$$
\mathrm{d} \phi_{\perp}(t, x)=\frac{1}{\varepsilon}\left[\Delta \phi_{\perp}(t, x)+a\left(t, \phi_{0}(t)\right) \phi_{\perp}(t, x)+b_{\perp}\left(t, \phi_{0}(t), \phi_{\perp}(t, x)\right)\right] \mathrm{d} t+\frac{\sigma}{\sqrt{\varepsilon}} \mathrm{d} W_{\perp}(t, x)
$$

admits, as in Subsection 3.2.2, a solution given by

$$
\begin{aligned}
\phi_{\perp}(t, \cdot)= & \frac{\sigma}{\sqrt{\varepsilon}} \int_{0}^{t} \mathrm{e}^{\alpha\left(t, t_{1}\right) / \varepsilon} \mathrm{e}^{\left[\left(t-t_{1}\right) / \varepsilon\right] \Delta} \mathrm{d} W\left(t_{1}, \cdot\right) \\
& +\frac{1}{\varepsilon} \int_{0}^{t} \mathrm{e}^{\alpha\left(t, t_{1}\right) / \varepsilon} \mathrm{e}^{\left[\left(t-t_{1}\right) / \varepsilon\right] \Delta} b_{\perp}\left(t_{1}, \phi_{0}\left(t_{1}\right), \phi_{\perp}\left(t_{1}, \cdot\right)\right) \mathrm{d} t_{1},
\end{aligned}
$$

where $\alpha\left(t, t_{1}\right)=\int_{t_{1}}^{t} a\left(u, \phi_{0}(u)\right) \mathrm{d} u$.

Proof of Theorem 2.9 The proof is virtually the same as the proof of Theorem 2.4, the only difference being that we use here the fact that $\phi_{0}(t)$ is bounded by a constant of order $T_{0}$, owing to the definition of $\mathcal{B}_{0}(h)$. Therefore, $a\left(t, \phi_{0}\right)$ is bounded above by a constant of order $T_{0}$. Since the largest eigenvalue of the Laplacian acting on mean-zero functions $\phi_{\perp}$ is equal to $-\pi^{2} / L^{2}$, taking $T_{0}$ small enough we obtain again a bound of the form (3.4) for the Lyapunov function $V=\left\|\phi_{\perp}\right\|_{H^{1}}^{2}$.

We now fix a realisation of $\phi_{\perp}(t)$. The difference $\psi_{0}(t)=\phi_{0}(t)-\bar{\phi}_{0}(t)$ satisfies the SDE

$$
\mathrm{d} \psi_{0}(t)=\frac{1}{\varepsilon}\left[\bar{a}\left(t, \bar{\phi}_{0}(t)\right) \psi_{0}(t)+\bar{b}\left(t, \psi_{0}(t)\right)\right] \mathrm{d} t+\frac{\sigma}{\sqrt{\varepsilon}} \mathrm{d} W_{0}(t)
$$

where

$$
\bar{a}\left(t, \bar{\phi}_{0}\right)=-2 \bar{\phi}_{0}-\partial_{\phi_{0}} b\left(t, \bar{\phi}_{0} e_{0}\right),
$$

and $\bar{b}\left(t, \psi_{0}(t)\right)$ denotes a non-linear term given by

$$
\begin{aligned}
\bar{b}\left(t, \psi_{0}\right)= & -\left(1+\frac{1}{2} \partial_{\phi_{0}} b\left(t, \bar{\phi}_{0} e_{0}+\theta \psi_{0} e_{0}\right)\right) \psi_{0}^{2} \\
& +b_{0}\left(t, \bar{\phi}_{0}(t)+\psi_{0}, \phi_{\perp}(t, \cdot)\right)-b_{0}\left(t, \bar{\phi}_{0}(t), \phi_{\perp}(t, \cdot)\right)
\end{aligned}
$$

for some $\theta \in(0,1)$. By (4.3), there is a constant $M>0$ such that $\bar{b}\left(t, \psi_{0}(t)\right)$ satisfies

$$
\left|\bar{b}\left(t, \psi_{0}(t)\right)\right| \leqslant M \psi_{0}(t)^{2}+2 M_{2}\left\|\phi_{\perp}\right\|_{H^{s}}^{2} \leqslant M \psi_{0}(t)^{2}+2 M_{2} h_{\perp}^{2} \quad \forall t<\tau_{\mathcal{B}_{\perp}\left(h_{\perp}\right)} .
$$

A solution of (4.4) is given by $\psi_{0}(t)=\psi_{0}^{0}(t)+\psi_{0}^{1}(t)$, where $\psi_{0}^{0}(t)$ is the solution of the linearisation of (4.4), and

$$
\psi_{0}^{1}(t)=\frac{1}{\varepsilon} \int_{-T_{0}}^{t} \mathrm{e}^{\bar{\alpha}\left(t, t_{1}\right) / \varepsilon} \bar{b}\left(t_{1}, \psi_{0}\left(t_{1}\right)\right) \mathrm{d} t_{1},
$$

where $\bar{\alpha}\left(t, t_{1}\right)=\int_{t_{1}}^{t} \bar{a}\left(t_{2}, \bar{\phi}_{0}\left(t_{2}\right)\right) \mathrm{d} t_{2}$.

Recall that we introduced a variance-related function $\zeta(t)$ satisfying (2.8). According to [5, Proposition 3.8], $\psi_{0}^{0}(t)$ is likely to remain in a strip of width proportional to $\sqrt{\zeta(t)}$. More precisely,

$$
\mathbb{P}\left\{\sup _{-T_{0} \leqslant t_{1} \leqslant t} \frac{\left|\psi_{0}^{0}\left(t_{1}\right)\right|}{\sqrt{\zeta\left(t_{1}\right)}} \geqslant h\right\} \leqslant C(t, \varepsilon) \exp \left\{-\frac{h^{2}}{2 \sigma^{2}}(1-\mathcal{O}(\varepsilon))\right\},
$$


where

$$
C(t, \varepsilon)=\frac{\left|\bar{\alpha}\left(t,-T_{0}\right)\right|}{\varepsilon^{2}}+2 .
$$

We now use this estimate to prove Theorem 2.10 ,

Proof of Theorem 2.10. For any decomposition $h=h_{0}+h_{1}$ with $h_{0}, h_{1}>0$, one has

$$
\begin{aligned}
& \mathbb{P}\left\{\tau_{\mathcal{B}_{0}(h)}<t \wedge \tau_{\mathcal{B}_{\perp}\left(h_{\perp}\right)}\right\}=\mathbb{P}\left\{\tau_{\mathcal{B}_{0}(h)}<t, \tau_{\mathcal{B}_{0}(h)}<\tau_{\mathcal{B}_{\perp}\left(h_{\perp}\right)}\right\} \\
& \leqslant \mathbb{P}\left\{\sup _{-T_{0} \leqslant t_{1} \leqslant t \wedge \tau_{\mathcal{B}_{0}(h)}} \frac{\left|\psi_{0}\left(t_{1}\right)\right|}{\sqrt{\zeta\left(t_{1}\right)}} \geqslant h, \tau_{\mathcal{B}_{0}(h)}<\tau_{\mathcal{B}_{\perp}\left(h_{\perp}\right)}\right\} \\
& \leqslant \mathbb{P}\left\{\sup _{-T_{0} \leqslant t_{1} \leqslant t} \frac{\left|\psi_{0}^{0}\left(t_{1}\right)\right|}{\sqrt{\zeta\left(t_{1}\right)}} \geqslant h_{0}\right\}+\mathbb{P}\left\{\sup _{-T_{0} \leqslant t_{1} \leqslant t \wedge \tau_{\mathcal{B}_{0}(h)}} \frac{\left|\psi_{0}^{1}\left(t_{1}\right)\right|}{\sqrt{\zeta\left(t_{1}\right)}} \geqslant h_{1}, \tau_{\mathcal{B}_{0}(h)}<\tau_{\mathcal{B}_{\perp}\left(h_{\perp}\right)}\right\}
\end{aligned}
$$

The first probability satisfies the bound (4.6), so that it remains to control the second one. By (4.5) and for all $t_{1} \leqslant t \wedge \tau_{\mathcal{B}_{0}(h)}<\tau_{\mathcal{B}_{\perp}\left(h_{\perp}\right)}$, as in [5, Proposition 3.10], we have the bound

$$
\begin{aligned}
\frac{\left|\psi_{0}^{1}\left(t_{1}\right)\right|}{\sqrt{\zeta\left(t_{1}\right)}} & \leqslant \frac{\left(M h^{2} \zeta\left(t_{1}\right)+M_{2} h_{\perp}^{2}\right)}{\sqrt{\zeta\left(t_{1}\right)}} \frac{1}{\varepsilon} \int_{-T_{0}}^{t_{1}} \mathrm{e}^{\bar{\alpha}\left(t_{1}, t_{2}\right) / \varepsilon} \mathrm{d} t_{2} \\
& \leqslant M h^{2} \hat{\zeta}(t)^{3 / 2}+M_{2} h_{\perp}^{2} \hat{\zeta}(t)^{1 / 2} .
\end{aligned}
$$

Choosing $h_{\perp}^{2} \leqslant \frac{M}{M_{2}} h^{2} \hat{\zeta}(t)$ and $h_{1}=$ const $h^{2} \hat{\zeta}(t)^{3 / 2}$, we get

$$
\mathbb{P}\left\{\sup _{-T_{0} \leqslant t_{1} \leqslant t \wedge \tau_{\mathcal{B}_{0}(h)}} \frac{\left|\psi_{0}^{1}\left(t_{1}\right)\right|}{\sqrt{\zeta\left(t_{1}\right)}} \geqslant h_{1}, \tau_{\mathcal{B}_{0}(h)}<\tau_{\mathcal{B}_{\perp}\left(h_{\perp}\right)}\right\}=0 .
$$

Therefore,

$$
\mathbb{P}\left\{\tau_{\mathcal{B}_{0}(h)}<t \wedge \tau_{\mathcal{B}_{\perp}\left(h_{\perp}\right)}\right\} \leqslant C(t, \varepsilon) \exp \left\{-\frac{h_{0}^{2}}{2 \sigma^{2}}(1-\mathcal{O}(\varepsilon))\right\} .
$$

We thus obtain the result by choosing $h_{0}=h-h_{1}=h-\mathcal{O}\left(h^{2} \hat{\zeta}(t)^{3 / 2}\right)=h\left(1-\mathcal{O}\left(h \hat{\zeta}(t)^{3 / 2}\right)\right)$. by

In weak noise regime, the probability of leaving either $\mathcal{B}_{0}(h)$ or $\mathcal{B}_{\perp}\left(h_{\perp}\right)$ before time $t$ is given

$$
\begin{aligned}
\mathbb{P} & \left\{\tau_{\mathcal{B}_{0}(h)} \wedge \tau_{\mathcal{B}_{\perp}\left(h_{\perp}\right)}<t\right\} \\
& =\mathbb{P}\left\{\tau_{\mathcal{B}_{0}(h)} \wedge \tau_{\mathcal{B}_{\perp}\left(h_{\perp}\right)}<t, \tau_{\mathcal{B}_{0}(h)}<\tau_{\mathcal{B}_{\perp}\left(h_{\perp}\right)}\right\}+\mathbb{P}\left\{\tau_{\mathcal{B}_{0}(h)} \wedge \tau_{\mathcal{B}_{\perp}\left(h_{\perp}\right)}<t, \tau_{\mathcal{B}_{\perp}\left(h_{\perp}\right)} \leqslant \tau_{\mathcal{B}_{0}(h)}\right\} \\
& =\mathbb{P}\left\{\tau_{\mathcal{B}_{0}(h)}<t, \tau_{\mathcal{B}_{0}(h)}<\tau_{\mathcal{B}_{\perp}\left(h_{\perp}\right)}\right\}+\mathbb{P}\left\{\tau_{\mathcal{B}_{\perp}\left(h_{\perp}\right)}<t, \tau_{\mathcal{B}_{\perp}\left(h_{\perp}\right)} \leqslant \tau_{\mathcal{B}_{0}(h)}\right\} \\
& =\mathbb{P}\left\{\tau_{\mathcal{B}_{0}(h)}<t \wedge \tau_{\mathcal{B}_{\perp}\left(h_{\perp}\right)}\right\}+\mathbb{P}\left\{\tau_{\mathcal{B}_{\perp}\left(h_{\perp}\right)}<t \wedge \tau_{\mathcal{B}_{0}(h)}\right\} .
\end{aligned}
$$

The first probability on the right-hand side is bounded by Theorem 2.10 and the second one by Theorem 2.9. Thus, we conclude that the behaviour of $\phi_{0}(t)$ in this regime does not differ much from the behaviour of the deterministic solution $\bar{\phi}_{0}(t)$ during the whole time interval $\left[-T_{0}, T_{0}\right]$.

However, in the strong-noise regime, the situation is different. We assume from now on that $\sigma \geqslant(\varepsilon \vee \delta)^{3 / 4}$, where Theorem 2.10 shows that sample paths are concentrated near the adiabatic solution tracking the stable potential well at $\phi_{+}^{*}$ up to times of order $-\sigma^{2 / 3}$. As time increases, it quickly becomes very unlikely not to reach and overcome the unstable solution $\hat{\phi}_{0}(t)$ tracking $\phi_{-}^{*}$. We notice that the linearisation of $f$ at $\hat{\phi}_{0}$ satisfies

$$
\hat{a}\left(t, \hat{\phi}_{0}(t)\right) \asymp(|t| \vee \sqrt{\delta \vee \varepsilon}) \asymp\left|\bar{a}\left(t, \bar{\phi}_{0}(t)\right)\right| \asymp \frac{1}{\zeta(t)} .
$$


In what follows, we prove Theorem 2.11, where the two terms on the right-hand side of (2.10) bound, respectively, the probability that $\phi_{0}$ does not reach $-d$ before time $t$, while staying below $\bar{\phi}_{0}+h \sqrt{\zeta}$, and the probability that $\phi_{0}$ crosses the level $\bar{\phi}_{0}+h \sqrt{\zeta}$ before time t.

Proof of Theorem 2.11 Let $h$ be such that $\bar{\phi}_{0}(t)+h \sqrt{\zeta(t)} \leqslant d$ for all $t \in\left[-c_{1} \sigma^{2 / 3}, c_{1} \sigma^{2 / 3}\right]$. We introduce the stopping times

$$
\begin{aligned}
& \tau_{+}=\inf \left\{t_{1} \in\left[-c_{1} \sigma^{2 / 3}, T_{0}\right]: \frac{\phi_{0}\left(t_{1}\right)-\bar{\phi}_{0}\left(t_{1}\right)}{\sqrt{\zeta\left(t_{1}\right)}}>h\right\}, \\
& \tau_{-}=\inf \left\{t_{1} \in\left[-c_{1} \sigma^{2 / 3}, T_{0}\right]: \phi_{0}\left(t_{1}\right)<-d\right\} .
\end{aligned}
$$

Then, the probability that $\phi_{0}$ does not reach $-d$ while $\phi_{\perp}$ remains in $\mathcal{B}_{\perp}\left(h_{\perp}\right)$ is given by

$$
\begin{aligned}
\mathbb{P}\left\{\tau_{-}\right. & \left.>t \wedge \tau_{\mathcal{B}_{\perp}\left(h_{\perp}\right)}\right\} \\
& =\mathbb{P}\left\{\tau_{-}>t \wedge \tau_{\mathcal{B}_{\perp}\left(h_{\perp}\right)}, \tau_{+} \leqslant t \wedge \tau_{\mathcal{B}_{\perp}\left(h_{\perp}\right)}\right\}+\mathbb{P}\left\{\tau_{-}>t \wedge \tau_{\mathcal{B}_{\perp}\left(h_{\perp}\right)}, \tau_{+}>t \wedge \tau_{\mathcal{B}_{\perp}\left(h_{\perp}\right)}\right\} \\
& \leqslant \mathbb{P}\left\{\tau_{+} \leqslant t \wedge \tau_{\mathcal{B}_{\perp}\left(h_{\perp}\right)}\right\}+\mathbb{P}\left\{\tau_{-} \wedge \tau_{+}>t \wedge \tau_{\mathcal{B}_{\perp}\left(h_{\perp}\right)}\right\}
\end{aligned}
$$

We estimate these two terms separately and the crucial term is the second one. Since we are going to use the Markov property and restart the process at certain times, we will use the notation $\mathbb{P}^{t_{0}, \phi_{0}}$ for the law of the process started at time $t_{0}$ in $\phi_{0}$ whenever necessary.

Proposition 4.1. Under the assumptions of Theorem 2.11 there exist constants $\kappa_{1}, M_{3}>0$ such that whenever $\left(-c_{1} \sigma^{2 / 3}, \phi_{0,0}\right) \in \mathcal{B}_{0}(h / 2)$, one has

$$
\mathbb{P}^{-c_{1} \sigma^{2 / 3}, \phi_{0,0}}\left\{\tau_{+} \leqslant t \wedge \tau_{\mathcal{B}_{\perp}\left(h_{\perp}\right)}\right\} \leqslant C(t, \varepsilon) \exp \left\{-\frac{\kappa_{1}}{2 \sigma^{2}}\left(h-M_{3} h_{\perp}^{2} \sqrt{\hat{\zeta}(t)}\right)^{2}\right\}
$$

for all $t \in\left[-c_{1} \sigma^{2 / 3}, T_{0}\right]$, where $C(t, \varepsilon)=\frac{\left|\bar{\alpha}\left(t,-c_{1} \sigma^{2 / 3}\right)\right|}{\varepsilon^{2}}+2$.

Proof: The solution of (4.4) is given by

$$
\psi_{0}(t)=\psi_{0}^{0}(t)+\frac{1}{\varepsilon} \int_{-c_{1} \sigma^{2 / 3}}^{t} \mathrm{e}^{\bar{\alpha}\left(t, t_{1}\right) / \varepsilon} \bar{b}\left(t_{1}, \psi_{0}\left(t_{1}\right)\right) \mathrm{d} t_{1} .
$$

We define a partition $-c_{1} \sigma^{2 / 3}=u_{0}<u_{1}<\cdots<u_{K}=t$ of $\left[-c_{1} \sigma^{2 / 3}, t\right]$ by

$$
\bar{\alpha}\left(u_{k}, u_{k-1}\right)=\varepsilon \quad \text { for } 1 \leqslant k \leqslant K=\left\lceil\frac{\bar{\alpha}\left(t,-c_{1} \sigma^{2 / 3}\right)}{\varepsilon}\right\rceil .
$$

We also introduce the notation $\rho_{k}=\frac{1}{2} h \sqrt{\zeta\left(u_{k}\right)}$. As shown in [5, Proposition 3.12], the Markov property implies

$$
\begin{aligned}
& \mathbb{P}^{-c_{1} \sigma^{2 / 3}, \phi_{0,0}}\left\{\tau_{+}<t \wedge \tau_{\mathcal{B}_{\perp}\left(h_{\perp}\right)}\right\}=\mathbb{P}^{-c_{1} \sigma^{2 / 3}, \phi_{0,0}}\left\{\sup _{-c_{1} \sigma^{2 / 3} \leqslant t_{1} \leqslant t \wedge \tau_{\mathcal{B}_{\perp}\left(h_{\perp}\right)}} \frac{\psi_{0}\left(t_{1}\right)}{\sqrt{\zeta\left(t_{1}\right)}}>h\right\} \\
& \leqslant \sum_{k=0}^{K-1} Q_{k}
\end{aligned}
$$


where

$$
\begin{aligned}
Q_{k}=\sup _{\psi_{0}\left(u_{k}\right) \leqslant \rho_{k}} & {\left[\mathbb{P}^{u_{k}, \psi_{0}\left(u_{k}\right)}\left\{\sup _{u_{k} \leqslant t_{1} \leqslant u_{k+1}} \frac{\psi_{0}\left(t_{1}\right)}{\sqrt{\zeta\left(t_{1}\right)}}>h\right\}\right.} \\
& \left.\left.+\mathbb{P}^{u_{k}, \psi_{0}\left(u_{k}\right)}\left\{\sup _{u_{k} \leqslant t_{1} \leqslant u_{k+1}} \frac{\psi_{0}\left(t_{1}\right)}{\sqrt{\zeta\left(t_{1}\right)}} \leqslant h, \psi_{0}\left(u_{k+1}\right)>\rho_{k+1}\right)\right\}\right] .
\end{aligned}
$$

For $h$ smaller than a constant of order 1 and $t_{1} \leqslant \tau_{\mathcal{B}_{\perp}\left(h_{\perp}\right)}$, (4.3) shows that $\bar{b}\left(t_{1}, \psi_{0}\left(t_{1}\right)\right)$ is bounded by $M_{2} h_{\perp}^{2}$. It follows that for any $t_{1} \in\left[u_{k}, u_{k+1}\right]$, one has

$$
\begin{aligned}
\psi_{0}^{1}\left(t_{1}\right) & \leqslant M_{2} h_{\perp}^{2} \int_{-c_{1} \sigma^{2 / 3}}^{t} \frac{1}{-\bar{a}\left(t_{1}, \bar{\phi}_{0}\left(t_{1}\right)\right)} \frac{-\bar{a}\left(t_{1}, \bar{\phi}_{0}\left(t_{1}\right)\right)}{\varepsilon} \mathrm{e}^{\bar{\alpha}\left(t, t_{1}\right) / \varepsilon} \mathrm{d} t_{1} \\
& \leqslant M_{2} h_{\perp}^{2} \sup _{u \in\left[u_{k}, u_{k+1}\right]} \frac{1}{\left|\bar{a}\left(u, \bar{\phi}_{0}(u)\right)\right|} .
\end{aligned}
$$

Therefore, there is a constant $M_{3}$ such that for any $t_{1} \in\left[u_{k}, u_{k+1}\right]$ one has

$$
\frac{\psi_{0}^{1}\left(t_{1}\right)}{\sqrt{\zeta\left(t_{1}\right)}} \leqslant M_{3} h_{\perp}^{2} \sqrt{\hat{\zeta}\left(u_{k+1}\right)} .
$$

Proceeding as in the proof of [5, Proposition 3.12], but with a shifted value of $h$, one obtains

$$
P_{k} \leqslant \exp \left\{-\frac{\kappa_{1}}{\sigma^{2}}\left(h-M_{3} h_{\perp}^{2} \sqrt{\hat{\zeta}\left(u_{k+1}\right)}\right)^{2}\right\}
$$

for some $\kappa_{1}>0$, which implies the claimed result.

The main part of the proof is contained in the following estimate, whose proof is very close in spirit to the proof of [5, Proposition 4.6], but with some changes due to the zero-mean part $\phi_{\perp}$ of the field.

Proposition 4.2. Under the assumptions of Theorem 2.11 there exists a choice of $c_{1}>0$ and constants $\bar{c}_{\perp}$ and $\kappa_{2}>0$ such that for $0<h_{\perp}<\bar{c}_{\perp} \sigma^{2 / 3}$, and all initial conditions $\phi_{0,0}$ in the interval $\left(-d, \bar{\phi}_{0}\left(-c_{1} \sigma^{2 / 3}\right)+h \sqrt{\zeta\left(-c_{1} \sigma^{2 / 3}\right)}\right]$, one has

$$
\begin{aligned}
& \mathbb{P}^{-c_{1} \sigma^{2 / 3}, \phi_{0,0}}\left\{\tau_{-} \wedge \tau_{+}>t \wedge \tau_{\mathcal{B}_{\perp}\left(h_{\perp}\right)}\right\} \\
& \quad=\mathbb{P}^{-c_{1} \sigma^{2 / 3}, \phi_{0,0}}\left\{-d<\phi_{0}\left(t_{1}\right) \leqslant \bar{\phi}_{0}\left(t_{1}\right)+h \sqrt{\zeta\left(t_{1}\right)} \forall t_{1} \in\left[-c_{1} \sigma^{2 / 3}, t \wedge \tau_{\mathcal{B}_{\perp}\left(h_{\perp}\right)}\right]\right\} \\
& \quad \leqslant \frac{3}{2} \exp \left\{-\kappa_{2} \frac{\hat{\alpha}\left(t,-c_{1} \sigma^{2 / 3}\right)}{\log \left(\sigma^{-1}\right) \varepsilon}\right\} .
\end{aligned}
$$

Proof: Let $\varrho \geqslant 1$ and define a partition $-c_{1} \sigma^{2 / 3}=u_{0}<u_{1}<\cdots<u_{K}=t$ by

$$
\hat{\alpha}\left(u_{k}, u_{k-1}\right)=\varrho \varepsilon \quad \text { for } 1 \leqslant k \leqslant K=\left\lceil\frac{\hat{\alpha}\left(t,-c_{1} \sigma^{2 / 3}\right)}{\varrho \varepsilon}\right\rceil .
$$

Writing

$$
Q_{k}=\sup _{\phi_{0}\left(u_{k}\right) \in\left(-d, \bar{\phi}_{0}\left(u_{k}\right)+h \sqrt{\zeta\left(u_{k}\right)}\right]} \mathbb{P}^{u_{k}, \phi_{0}\left(u_{k}\right)}\left\{-d<\phi_{0} \leqslant \bar{\phi}_{0}\left(t_{1}\right)+h \sqrt{\zeta\left(t_{1}\right)} \forall t_{1} \in\left[u_{k}, u_{k+1}\right]\right\}
$$


we have, by the Markov property,

$$
\begin{aligned}
\mathbb{P}^{-c_{1} \sigma^{2 / 3}, \phi_{0,0}}\left\{-d<\phi_{0}\left(t_{1}\right) \leqslant \bar{\phi}_{0}\left(t_{1}\right)+h \sqrt{\zeta\left(t_{1}\right)} \forall t_{1} \in\left[-c_{1} \sigma^{2 / 3}, t\right]\right\} \\
=\mathbb{E}^{-c_{1} \sigma^{2 / 3}, \phi_{0,0}}\left\{1_{\left\{-d<\phi_{0}\left(t_{1}\right) \leqslant \bar{\phi}_{0}\left(t_{1}\right)+h \sqrt{\zeta\left(t_{1}\right)} \forall t_{1} \in\left[-c_{1} \sigma^{2 / 3}, u_{K-1}\right]\right\}}\right. \\
\left.\quad \times \mathbb{P}^{u_{K-1}, \phi_{0}\left(u_{K-1}\right)}\left\{-d<\phi_{0}\left(t_{1}\right) \leqslant \bar{\phi}_{0}\left(t_{1}\right)+h \sqrt{\zeta\left(t_{1}\right)} \forall t_{1} \in\left[u_{K-1}, u_{K}\right]\right\}\right\} \\
\leqslant Q_{K-1} \mathbb{P}^{-c_{1} \sigma^{2 / 3}, \phi_{0,0}}\left\{-d<\phi_{0}\left(t_{1}\right) \leqslant \bar{\phi}_{0}\left(t_{1}\right)+h \sqrt{\zeta\left(t_{1}\right)} \forall t_{1} \in\left[-c_{1} \sigma^{2 / 3}, u_{K-1}\right]\right\} \\
\leqslant \cdots \leqslant \prod_{k=0}^{K-1} Q_{k} .
\end{aligned}
$$

Our plan is to show that for an appropriate choice of $\varrho, Q_{k}$ is bounded away from 1 for $k=$ $0, \ldots, K-1$. In order to estimate $Q_{k}$ we shall distinguish three cases corresponding to $\phi_{0}$ crossing the levels $\bar{\phi}_{0}$ and $\hat{\phi}_{0}$ before reaching $-d$. We set

$$
\bar{M}_{k}=M_{2} h_{\perp}^{2} \sup _{u \in\left[u_{k}, u_{k+1}\right]} \frac{1}{\left|\bar{a}\left(u, \bar{\phi}_{0}(u)\right)\right|}, \quad \widehat{M}_{k}=M_{2} h_{\perp}^{2} \sup _{u \in\left[u_{k}, u_{k+1}\right]} \frac{1}{\hat{a}\left(u, \hat{\phi}_{0}(u)\right)},
$$

and introduce a further subdivision $u_{k}<\tilde{u}_{k, 1}<\tilde{u}_{k, 2}<u_{k+1}$ defined by

$$
\hat{\alpha}\left(\tilde{u}_{k, 1}, u_{k}\right)=\frac{1}{3} \varrho \varepsilon, \quad \hat{\alpha}\left(\tilde{u}_{k, 2}, u_{k}\right)=\frac{2}{3} \varrho \varepsilon .
$$

Define the stopping times

$$
\begin{aligned}
& \tau_{k, 1}=\inf \left\{t_{1} \in\left[u_{k}, \tilde{u}_{k, 1}\right]: \phi_{0}\left(t_{1}\right) \leqslant \bar{\phi}_{0}\left(t_{1}\right)+\bar{M}_{k}\right\}, \\
& \tau_{k, 2}=\inf \left\{t_{1} \in\left[u_{k}, \tilde{u}_{k, 2}\right]: \phi_{0}\left(t_{1}\right) \leqslant \hat{\phi}_{0}\left(t_{1}\right)+\widehat{M}_{k}\right\} .
\end{aligned}
$$

Then we can write

$$
\begin{aligned}
\mathbb{P}^{u_{k}, \phi_{0}\left(u_{k}\right)}\left\{-d<\phi_{0}\left(t_{1}\right) \leqslant \bar{\phi}_{0}\left(t_{1}\right)+h \sqrt{\zeta\left(t_{1}\right)} \forall t_{1} \in\left[u_{k}, u_{k+1}\right]\right\} \\
\leqslant \mathbb{P}^{u_{k}, \phi_{0}\left(u_{k}\right)}\left\{\bar{\phi}_{0}\left(t_{1}\right)+\bar{M}_{k}<\phi_{0}\left(t_{1}\right) \leqslant \bar{\phi}_{0}\left(t_{1}\right)+h \sqrt{\zeta\left(t_{1}\right)} \forall t_{1} \in\left[u_{k}, \tilde{u}_{k, 1}\right]\right\} \\
+\mathbb{E}^{u_{k}, \phi_{0}\left(u_{k}\right)}\left\{1_{\left\{\tau_{k, 1}<\tilde{u}_{k, 1}\right\}}\right. \\
\left.\quad \times \mathbb{P}^{\tau_{k, 1}, \phi_{0}\left(\tau_{k, 1}\right)}\left\{-d<\phi_{0}\left(t_{1}\right) \leqslant \bar{\phi}_{0}\left(t_{1}\right)+h \sqrt{\zeta\left(t_{1}\right)} \forall t_{1} \in\left[\tau_{k, 1}, u_{k+1}\right]\right\}\right\} .
\end{aligned}
$$

We start by bounding the first term on the right-hand side. Let

$$
\psi_{0}^{(k)}\left(t_{1}\right)=\psi_{0}\left(u_{k}\right) \mathrm{e}^{\bar{\alpha}\left(t_{1}, u_{k}\right) / \varepsilon}+\frac{\sigma}{\sqrt{\varepsilon}} \int_{u_{k}}^{t_{1}} \mathrm{e}^{\bar{\alpha}\left(t_{1}, v\right) / \varepsilon} \mathrm{d} W_{0}(v)
$$

be the solution of the equation linearised around $\bar{\phi}_{0}(t)$, starting in $\psi_{0}\left(u_{k}\right)=\phi_{0}\left(u_{k}\right)-\bar{\phi}_{0}\left(u_{k}\right)$. Then in follows from (4.8) that

$$
\psi_{0}(t) \leqslant \psi_{0}^{(k)}(t)+\bar{M}_{k} \quad \forall t_{1} \in\left[u_{k}, u_{k+1}\right] .
$$


Note that $\psi_{0}^{(k)}\left(\tilde{u}_{k, 1}\right)$ is a normal random variable with parameters

$$
\begin{aligned}
\mathbb{E}\left[\psi_{0}^{(k)}\left(\tilde{u}_{k, 1}\right)\right] & =\psi_{0}\left(u_{k}\right) \mathrm{e}^{\bar{\alpha}\left(\tilde{u}_{k, 1}, u_{k}\right) / \varepsilon} \leqslant \psi_{0}\left(u_{k}\right) \mathrm{e}^{-\varrho / 3 R} \\
\operatorname{Var}\left(\psi_{0}^{(k)}\left(\tilde{u}_{k, 1}\right)\right) & =\frac{\sigma^{2}}{\varepsilon} \int_{u_{k}}^{\tilde{u}_{k, 1}} \mathrm{e}^{2 \bar{\alpha}\left(t_{1}, v\right) / \varepsilon} \mathrm{d} v \\
& \geqslant \frac{\sigma^{2}}{2} \inf _{u_{k} \leqslant t_{1} \leqslant u_{k+1}} \frac{1}{\left|\bar{a}\left(u, \bar{\phi}_{0}(u)\right)\right|}\left[1-\mathrm{e}^{-2 \varrho / 3 R}\right],
\end{aligned}
$$

where $R>0$ is a constant such that $\hat{a}\left(t_{1}, \hat{\phi}_{0}\left(t_{1}\right)\right) \leqslant R\left|\bar{a}\left(t_{1}, \bar{\phi}_{0}\left(t_{1}\right)\right)\right|$ for all $t_{1} \in\left[-c_{1} \sigma^{2 / 3}, t\right]$. Then André's reflection principle shows that the first term on the right-hand side of (4.10) is bounded above by

$$
\begin{aligned}
\mathbb{P}^{u_{k}, \phi_{0}\left(u_{k}\right)}\left\{\psi_{0}^{(k)}\left(t_{1}\right)>0 \forall t_{1} \in\left[u_{k}, \tilde{u}_{k, 1}\right]\right\} & =1-2 \mathbb{P}^{u_{k}, \phi_{0}\left(u_{k}\right)}\left\{\psi_{0}^{(k)}\left(t_{1}\right) \leqslant 0\right\} \\
& =2 \mathbb{P}^{u_{k}, \phi_{0}\left(u_{k}\right)}\left\{\psi_{0}^{(k)}\left(t_{1}\right)>0\right\}-1 \\
& \leqslant \frac{2}{\sqrt{\pi}} \frac{h}{\sigma} C_{1}(k) \frac{\mathrm{e}^{-\varrho / 3 R}}{\sqrt{1-\mathrm{e}^{-2 \varrho / 3 R}}},
\end{aligned}
$$

where

$$
C_{1}(k)=\sup _{u_{k} \leqslant t_{1} \leqslant u_{k+1}} \sqrt{\left|\bar{a}\left(u_{k}, \bar{\phi}_{0}\right)\right|} \sqrt{\zeta\left(u_{k}\right)}
$$

is a constant of order 1 , owing to 4.7.

In order to bound the second term on the right-hand side of 4.10), we set set $\varphi_{0}(t)=$ $\phi_{0}(t)-\hat{\phi}_{0}(t)$, where we recall that $\hat{\phi}_{0}(t)$ is the deterministic solution tracking $\phi_{-}^{*}$. Observe that if $\tau_{k, 1}<\tilde{u}_{k, 1}$, we also have

$$
\begin{aligned}
& \mathbb{P}^{\tau_{k, 1}, \varphi_{0}\left(\tau_{k, 1}\right)}\left\{-d<\hat{\phi}_{0}\left(t_{1}\right)+\varphi_{0}\left(t_{1}\right) \leqslant \bar{\phi}_{0}\left(t_{1}\right)+h \sqrt{\zeta\left(t_{1}\right)} \forall t_{1} \in\left[\tau_{k, 1}, u_{k+1}\right]\right\} \\
& \leqslant \mathbb{P}^{\tau_{k, 1}, \varphi_{0}\left(\tau_{k, 1}\right)}\left\{\widehat{M}_{k}<\varphi_{0}\left(t_{1}\right) \leqslant \bar{\phi}_{0}\left(t_{1}\right)-\hat{\phi}_{0}\left(t_{1}\right)+h \sqrt{\zeta\left(t_{1}\right)} \forall t_{1} \in\left[\tau_{k, 1}, \tilde{u}_{k, 2}\right]\right\} \\
& +\mathbb{E}^{u_{k}, \varphi_{0}\left(u_{k}\right)}\left\{1_{\left\{\tau_{k, 2}<\tilde{u}_{k, 2}\right\}}\right. \\
& \left.\quad \times \mathbb{P}^{\tau_{k, 2}, \varphi_{0}\left(\tau_{k, 2}\right)}\left\{-d<\hat{\phi}_{0}\left(t_{1}\right)+\varphi_{0}\left(t_{1}\right) \leqslant \bar{\phi}_{0}\left(t_{1}\right)+h \sqrt{\zeta\left(t_{1}\right)} \forall t_{1} \in\left[\tau_{k, 2}, u_{k+1}\right]\right\}\right\} .
\end{aligned}
$$

To bound the first term on the right-hand side, we introduce the linear process

$$
\varphi_{0}^{(k)}\left(t_{1}\right)=\varphi_{0}\left(\tilde{u}_{k, 1}\right) \mathrm{e}^{\hat{\alpha}\left(t_{1}, \tilde{u}_{k, 1}\right) / \varepsilon}+\frac{\sigma}{\sqrt{\varepsilon}} \int_{\tilde{u}_{k, 1}}^{t_{1}} \mathrm{e}^{\hat{\alpha}\left(t_{1}, v\right) / \varepsilon} \mathrm{d} W_{0}(v)
$$

which satisfies

$$
\varphi_{0}(t) \leqslant \varphi_{0}^{(k)}(t)+\widehat{M}_{k} \quad \forall t_{1} \in\left[\tilde{u}_{k, 1}, u_{k+1}\right] .
$$

Then we have the estimates

$$
\begin{aligned}
\mathbb{E}\left[\varphi_{0}^{(k)}\left(\tilde{u}_{k, 2}\right)\right] & =\varphi_{0}\left(\tau_{k, 1}\right) \mathrm{e}^{\hat{\alpha}\left(\tilde{u}_{k, 2}, \tau_{k, 1}\right) / \varepsilon} \\
& \leqslant\left[\bar{\phi}_{0}\left(\tau_{k, 1}\right)+\bar{M}_{k}-\hat{\phi}_{0}\left(\tau_{k, 1}\right)\right] \mathrm{e}^{\varrho / 3}, \\
\mathrm{e}^{-2 \hat{\alpha}\left(\tilde{u}_{k, 2}, \tau_{k, 1}\right) / \varepsilon} \operatorname{Var}\left(\varphi_{0}^{(k)}\left(\tilde{u}_{k, 2}\right)\right) & \geqslant \inf _{u_{k} \leqslant t_{1} \leqslant u_{k+1}} \frac{\sigma^{2}}{2 \hat{a}\left(t_{1}, \hat{\phi}_{0}\left(t_{1}\right)\right)}\left[1-\mathrm{e}^{-2 \varrho / 3}\right] .
\end{aligned}
$$


The first term on the right-hand side of (4.12) can then be bounded by

$$
\frac{2}{\sqrt{\pi}} \frac{1}{\sigma} C_{2}(k) \frac{1}{\sqrt{1-\mathrm{e}^{-2 \varrho / 3}}},
$$

where

$$
C_{2}(k)=\sup _{u_{k} \leqslant t_{1} \leqslant u_{k+1}} \sqrt{\hat{a}\left(t_{1}, \hat{\phi}_{0}\left(t_{1}\right)\right)} \sup _{u_{k} \leqslant t_{1} \leqslant u_{k+1}}\left(\bar{\phi}_{0}\left(t_{1}\right)+\bar{M}_{k}-\hat{\phi}_{0}\left(t_{1}\right)\right) .
$$

Finally, in order to estimate the second summand in (4.12), we use the end point estimate

$$
\begin{aligned}
& \mathbb{P}^{\tau_{k, 2}, \varphi_{0}\left(\tau_{k, 2}\right)}\left\{-d<\hat{\phi}_{0}\left(t_{1}\right)+\varphi_{0}\left(t_{1}\right) \leqslant \bar{\phi}_{0}\left(t_{1}\right)+h \sqrt{\zeta\left(t_{1}\right)} \forall t_{1} \in\left[\tau_{k, 2}, u_{k+1}\right]\right\} \\
& \leqslant \mathbb{P}^{\tau_{k, 2}, \varphi_{0}\left(\tau_{k, 2}\right)}\left\{-d<\hat{\phi}_{0}\left(u_{k+1}\right)+\varphi_{0}\left(u_{k+1}\right)\right\} \\
& \leqslant \frac{1}{2}+\frac{1}{\sqrt{\pi}} \frac{1}{\sigma} C_{3}(k)\left[d+\hat{\phi}_{0}\left(u_{k+1}\right)+\widehat{M}_{k}\right] \frac{\mathrm{e}^{-\varrho / 3}}{\sqrt{1-\mathrm{e}^{-2 \varrho / 3}}}
\end{aligned}
$$

where

$$
C_{3}(k)=\sup _{u_{k} \leqslant t_{1} \leqslant u_{k+1}} \sqrt{\hat{a}\left(t_{1}, \hat{\phi}_{0}\left(t_{1}\right)\right)} .
$$

Summing (4.11), (4.13) and (4.14) we get the existence of a constant $C_{0}>0$ such that

$$
Q_{k} \leqslant \frac{1}{2}+C_{0}\left[\frac{h}{\sigma} \mathrm{e}^{-\varrho / 3 R} C_{1}(k)+\frac{1}{\sigma} C_{2}(k)+\frac{1}{\sigma} \mathrm{e}^{-\varrho / 3} C_{3}(k)\left(1+\widehat{M}_{k}\right)\right] .
$$

Since

$$
\begin{aligned}
\left|t_{1}\right| & \leqslant c_{1} \sigma^{2 / 3}, \\
\hat{a}\left(t_{1}, \hat{\phi}_{0}\left(t_{1}\right)\right) & \asymp\left|t_{1}\right| \vee \sqrt{\delta \vee \varepsilon}, \\
\bar{\phi}_{0}\left(t_{1}\right)-\hat{\phi}_{0}\left(t_{1}\right) & \leqslant \bar{c}_{1}\left|t_{1}\right|,
\end{aligned}
$$

where $\bar{c}_{1}$ is proportional to $c_{1}$ and $\varrho \geqslant 1$, there exists another constant $C_{4}$ such that

$$
Q_{k} \leqslant \frac{1}{2}+C_{4}\left[\frac{h}{\sigma} \mathrm{e}^{-\varrho / 3 R}+\bar{c}_{1}^{3 / 2}+\frac{h_{\perp}^{2}}{\sigma^{4 / 3}}\left(1+\mathrm{e}^{-\varrho / 3}\right)+\frac{1}{\sigma^{2 / 3}} \mathrm{e}^{-\varrho / 3}\right] .
$$

Choosing $h_{\perp} \leqslant \bar{c}_{\perp} \sigma^{2 / 3}$ we get

$$
Q_{k} \leqslant \frac{1}{2}+C_{4}\left[\frac{h}{\sigma} \mathrm{e}^{-\varrho / 3 R}+\bar{c}_{1}^{3 / 2}+2 \bar{c}_{\perp}^{2}+\frac{1}{\sigma^{2 / 3}} \mathrm{e}^{-\varrho / 3}\right] .
$$

For $c_{1}$ such that $\bar{c}_{1}^{3 / 2}=2 \bar{c}_{\perp}^{2}=\frac{1}{24 C_{4}}$ and

$$
\varrho=3 R \log \left(36 C_{4} \frac{h}{\sigma}\right) \vee 3 \log \left(\frac{18 C_{4}}{c_{1} \sigma^{2 / 3}}\right) \vee 1,
$$

$Q_{k}$ is bounded by $\frac{2}{3}$ for $k=0, \ldots, K-1$. We conclude that with this choice of $\varrho$, we have

$$
\begin{gathered}
\mathbb{P}^{-c_{1} \sigma^{2 / 3}, \phi_{0}}\left\{-d<\phi_{0}\left(t_{1}\right) \leqslant \bar{\phi}_{0}\left(t_{1}\right)+h \sqrt{\zeta\left(t_{1}\right)} \forall t_{1} \in\left[-c_{1} \sigma^{2 / 3}, t\right]\right\} \\
\leqslant\left(\frac{2}{3}\right)^{K-1}=\frac{3}{2} \exp \left\{-K \log \left(\frac{3}{2}\right)\right\}
\end{gathered}
$$

which yields the claimed result, owing to our choice (4.9) of $K$, and the fact that $\varrho$ has order $\log \left(\sigma^{-1}\right)$. 
The conclusion of Theorem 2.11 now follows immediately by combining the last two propositions.

Proof of Proposition 2.13 We introduce the stopping times

$$
\begin{aligned}
& \tau_{+}=\inf \left\{t_{1} \in\left[t_{0}, t_{0}+\tilde{c} \varepsilon\right]: \phi_{0}\left(t_{1}\right)>-d+\rho\right\}, \\
& \tau_{-}=\inf \left\{t_{1} \in\left[t_{0}, t_{0}+\tilde{c} \varepsilon\right]: \phi_{0}\left(t_{1}\right)<-d_{0}\right\},
\end{aligned}
$$

and the process

$$
\tilde{\phi}_{0}(t)=-d-\frac{1}{\varepsilon} f_{0}\left(t-t_{0}\right)+\frac{\sigma}{\sqrt{\varepsilon}} W_{t-t_{0}} .
$$

Taking the constant $M$ in the statement of the proposition equal to $M_{2}$, one can check, in a similar way as before, that $\phi_{0}\left(t_{1}\right) \leqslant \tilde{\phi}_{0}\left(t_{1}\right)$ for all $t_{1} \leqslant \tau_{-} \wedge \tau_{+} \wedge \tau_{\mathcal{B}_{\perp}\left(h_{\perp}\right)}$. Now we observe that

$$
\begin{aligned}
\mathbb{P}\left\{\tilde{\phi}_{0}(t)\right. & \left.\geqslant-d_{0} \forall t \in\left[t_{0}, t_{0}+\tilde{c} \varepsilon\right]\right\} \\
\leqslant & \mathbb{P}\left\{\sup _{t \in\left[t_{0}, t_{0}+\tilde{c} \varepsilon\right]}\left[\tilde{\phi}_{0}(t)+\frac{1}{\varepsilon} f_{0}\left(t-t_{0}\right)\right]>-d+\rho\right\} \\
& +\mathbb{P}\left\{-d_{0} \leqslant \tilde{\phi}_{0}(t) \leqslant-d+\rho-\frac{1}{\varepsilon} f_{0}\left(t-t_{0}\right) \forall t \in\left[t_{0}, t_{0}+\tilde{c} \varepsilon\right]\right\} .
\end{aligned}
$$

The second term on the right-hand side vanishes as soon as we take $\tilde{c}>\left(d_{0}-d+\rho\right) / f_{0}$, while the first one is equal to

$$
\mathbb{P}\left\{\sup _{t \in\left[t_{0}, t_{0}+\tilde{c} \varepsilon\right]} \frac{\sigma}{\sqrt{\varepsilon}} W_{t-t_{0}}>\rho\right\} \leqslant \mathrm{e}^{-\rho^{2} /\left(2 \tilde{c} \sigma^{2}\right)}
$$

by a Bernstein-type inequality. Now we note that for any $t \in\left[t_{0}, t_{0}+\tilde{c} \varepsilon\right]$, we have

$$
\begin{aligned}
\mathbb{P}\left\{\tau_{-}>t\right\} & =\mathbb{P}\left\{\tau_{-}>t, t \leqslant \tau_{-} \wedge \tau_{+} \wedge \tau_{\mathcal{B}_{\perp}\left(h_{\perp}\right)}\right\}+\mathbb{P}\left\{\tau_{+} \wedge \tau_{\mathcal{B}_{\perp}\left(h_{\perp}\right)}<t<\tau_{-}\right\} \\
& \leqslant \mathbb{P}\left\{\tilde{\phi}_{0}(t) \geqslant-d_{0} \forall t \in\left[t_{0}, t_{0}+\tilde{c} \varepsilon\right]\right\}+\mathbb{P}\left\{\tau_{+} \wedge \tau_{\mathcal{B}_{\perp}\left(h_{\perp}\right)}<t \wedge \tau_{-}\right\} .
\end{aligned}
$$

We have already shown that the first term on the right-hand side is exponentially small, and the second term can be controlled as in the preceding results.

\section{A Some useful inequalities in Sobolev spaces}

Given $\psi \in L^{2}(\mathbb{T})$, Sobolev's inequality states that given any $p \geqslant 2$, for any $s>\frac{1}{2}-\frac{1}{p}$, there exists a finite constant $C_{\mathrm{Sob}}(s, p)$ such that

$$
\|\psi\|_{L^{p}} \leqslant C_{\text {Sob }}(s, p)\|\psi\|_{H^{s}} .
$$

The following estimate on products in Sobolev spaces applies to the case $s>\frac{1}{2}$. A concise proof can be found in [10, Théorème 7].

Lemma A.1 (Products in Sobolev Spaces). If $s>\frac{1}{2}$ then there is a bilinear application

$$
\begin{aligned}
H^{s}(\mathbb{T}) \times H^{s}(\mathbb{T}) & \longrightarrow H^{s}(\mathbb{T}) \\
(\psi, \phi) & \longmapsto \psi \phi,
\end{aligned}
$$

which coincides with the pointwise product and satisfies the estimate

$$
\|\psi \phi\|_{H^{s}(\mathbb{T})} \leqslant C\|\psi\|_{H^{s}(\mathbb{T})}\|\phi\|_{H^{s}(\mathbb{T})}
$$

for some finite constant $C=C(s)$. 
While the above result does not hold if $s \leqslant \frac{1}{2}$, we have the following consequence of Young's inequality, a proof of which can be found, for instance, in [8, Lemma 4.3].

Lemma A.2 (Young-type inequality). Let $r, s, t \in\left(0, \frac{1}{2}\right)$ be such that $t<r+s-\frac{1}{2}$. Then there exists a finite constant $C=C(r, s, t)$ such that

$$
\|\psi * \phi\|_{H^{t}} \leqslant C\|\psi\|_{H^{r}}\|\phi\|_{H^{s}}<\infty .
$$

\section{References}

[1] Hassan Alkhayuon, Rebecca C. Tyson, and Sebastian Wieczorek. Phase-sensitive tipping: How cyclic ecosystems respond to contemporary climate. arXiv:2101.12107.

[2] Roberto Benzi, Alfonso Sutera, and Angelo Vulpiani. The mechanism of stochastic resonance. $J$. Phys. A, 14(11):L453-L457, 1981.

[3] Nils Berglund. An Eyring-Kramers law for slowly oscillating bistable diffusions. Preprint $\operatorname{arXiv/2007.08443,2020.}$

[4] Nils Berglund and Barbara Gentz. Pathwise description of dynamic pitchfork bifurcations with additive noise. Probab. Theory Related Fields, 122(3):341-388, 2002.

[5] Nils Berglund and Barbara Gentz. A sample-paths approach to noise-induced synchronization: stochastic resonance in a double-well potential. Ann. Appl. Probab., 12(4):1419-1470, 2002.

[6] Nils Berglund and Barbara Gentz. Geometric singular perturbation theory for stochastic differential equations. J. Differential Equations, 191:1-54, 2003.

[7] Nils Berglund and Barbara Gentz. Noise-induced phenomena in slow-fast dynamical systems. A sample-paths approach. Probability and its Applications (New York). Springer-Verlag London, Ltd., London, 2006.

[8] Nils Berglund and Barbara Gentz. Sharp estimates for metastable lifetimes in parabolic SPDEs: Kramers' law and beyond. Electron. J. Probab., 18:no. 24, 58, 2013.

[9] Nils Berglund and Barbara Gentz. On the Noise-Induced Passage through an Unstable Periodic Orbit II: General Case. SIAM J. Math. Anal., 46(1):310-352, 2014.

[10] Gérard Bourdaud. Le calcul symbolique dans certaines algèbres de type Sobolev. In Recent developments in fractals and related fields, Appl. Numer. Harmon. Anal., pages 131-144. Birkhäuser Boston, Boston, MA, 2010.

[11] Gerard Bourdaud and Yves Meyer. Fonctions qui opèrent sur les espaces de Sobolev. Journal of Functional Analysis, 97(2):351-360, 1991.

[12] I. Eisenman and J. S. Wettlaufer. Nonlinear threshold behavior during the loss of Arctic sea ice. PNAS, 106(1):28-32, 2009.

[13] Neil Fenichel. Geometric singular perturbation theory for ordinary differential equations. J. Differential Equations, 31(1):53-98, 1979.

[14] Ronald F. Fox. Stochastic resonance in a double well. Phys. Rev. A, 39:4148-4153, 1989.

[15] L. Gammaitoni, E. Menichella-Saetta, S. Santucci, F. Marchesoni, and C. Presilla. Periodically time-modulated bistable systems: Stochastic resonance. Phys. Rev. A, 40:2114-2119, 1989.

[16] Luca Gammaitoni, Peter Hänggi, Peter Jung, and Fabio Marchesoni. Stochastic resonance. Rev. Mod. Phys., 70:223-287, 1998.

[17] Manuel V. Gnann, Christian Kuehn, and Anne Pein. Towards sample path estimates for fast-slow stochastic partial differential equations. European Journal of Applied Mathematics, 30(5):1004-1024, 2019. 
[18] Peter Hänggi. Stochastic resonance in biology: How noise can enhance detection of weak signals and help improve biological information processing. Chemphyschem, 3(3):285-290, 2002.

[19] Samuel Herrmann, Peter Imkeller, Ilya Pavlyukevich, and Dierk Peithmann. Stochastic resonance. A mathematical approach in the small noise limit. American Mathematical Society, Providence, RI, 2014.

[20] G. Jetschke. On the equivalence of different approaches to stochastic partial differential equations. Math. Nachr., 128:315-329, 1986.

[21] Peter Jung and Peter Hänggi. Amplification of small signals via stochastic resonance. Phys. Rev. A, 44:8032-8042, 1991.

[22] C.B. Muratov, E. Vanden-Eijnden, and W. E. Self-induced stochastic resonance in excitable systems. Physica D, 210:227-240, 2005.

[23] C. Nicolis and G. Nicolis. Stochastic aspects of climatic transitions-additive fluctuations. Tellus, 33(3):225-234, 1981.

[24] A. N. Tihonov. Systems of differential equations containing small parameters in the derivatives. Mat. Sbornik N. S., 31:575-586, 1952.

[25] P. Vélez-Belchí, A. Alvarez, P. Colet, J. Tintoré, and R. L. Haney. Stochastic resonance in the thermohaline circulation. Geophysical Research Letters, 28(10):2053-2056, 2001.

[26] Kurt Wiesenfeld and Fernan Jaramillo. Minireview of stochastic resonance. Chaos, 8:539-548, 1998.

[27] Kurt Wiesenfeld and Frank Moss. Stochastic resonance and the benefits of noise: From ice ages to crayfish and SQUIDs. Nature, 373:33-36, 1995. 


\section{Contents}

1 Introduction 1

2 Main results

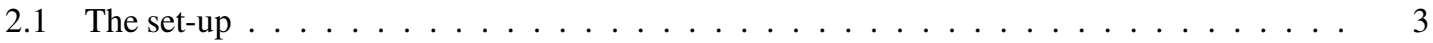

2.2 The stable case . . . . . . . . . . . . . . . . . . . . . . 4

2.3 Bifurcations and avoided bifurcations . . . . . . . . . . . . . . . 6

2.4 Discussion . . . . . . . . . . . . . . . . . . . . 11

3 Proofs: the stable case $\quad 13$

3.1 Deterministic case . . . . . . . . . . . . . . . . . . . . . 13

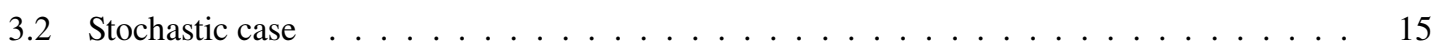

4 Proofs: bifurcations $\quad 21$

4.1 Deterministic case . . . . . . . . . . . . . . . . . . . . . . . . . . . . . . . . . .

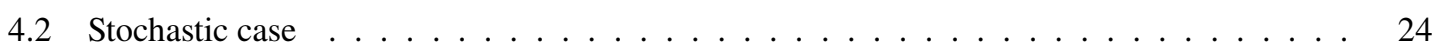

A Some useful inequalities in Sobolev spaces 31

Institut Denis Poisson (IDP)

Université d'Orléans, Université de Tours, CNRS - UMR 7013

Bâtiment de Mathématiques, B.P. 6759

45067 Orléans Cedex 2, France

E-mail addresses: nils.berglund@univ-orleans.fr, rita.nader@univ-orleans.fr 